\title{
MATERNAL SENSITIVITY AND INFANT STRESS SYSTEM COORDINATION AND FLEXIBILITY
}

by

\author{
Brittany Jamieson
}

Bachelor of Arts and Science, University of Guelph, 2013

A thesis presented to Ryerson University

\author{
In partial fulfillment of the \\ requirements for the degree of \\ Master of Arts \\ In the Program of Psychology \\ Toronto, Ontario, Canada, 2016 \\ C (Brittany Jamieson) 2016
}




\section{AUTHOR'S DECLARATION}

I hereby declare that I am the sole author of this thesis. This is a true copy of the thesis, including any required final revisions, as accepted by my examiners.

I authorize Ryerson University to lend this thesis to other institutions or individuals for the purpose of scholarly research.

I further authorize Ryerson University to reproduce this thesis by photocopying or by other means, in total or in part, at the request of other institutions or individuals for the purpose of scholarly research.

I understand that my thesis may be made electronically available to the public. 


\title{
MATERNAL SENSITIVITY AND INFANT STRESS SYSTEM \\ COORDINATION AND FLEXIBILITY
}

Master of Arts, 2016

Brittany Jamieson

Psychology

Ryerson University

\begin{abstract}
Previous research has assessed the relationship between maternal sensitivity and infant hypothalamic-pituitary-adrenocortical [HPA] axis function, yet neglected additional stress systems. Using a multi-system method (HPA measured via cortisol and sympathetic nervous system via salivary alpha-amylase; sAA), we assessed the relationship between maternal sensitivity and infant stress system coordination and flexibility in response to acute stress. A community sample of 125 mother-infant dyads participated in a toy frustration (age 15 months) and separation procedure (age 16 months). Maternal sensitivity was measured via naturalistic observation. Multilevel-modeling analyses found that maternal sensitivity moderates the relationship between infant sAA and cortisol basal activity and reactivity, such that systems were coordinated at higher, but not lower, levels of sensitivity. SAA output was greater in response to separation compared to frustration, though sensitivity did not moderate this variability. Findings suggest that the quality of early caregiving relationships is important for the development of coordinated stress physiology.
\end{abstract}

Keywords: maternal sensitivity, salivary-alpha amylase, cortisol, coordination, flexibility, infants 


\section{Acknowledgements}

This research was supported by funds from the Social Sciences and Humanities Research

Council, Ryerson University and The Institute for Stress and Well-being Research. 


\section{TABLE OF CONTENTS}

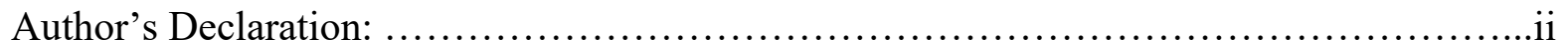

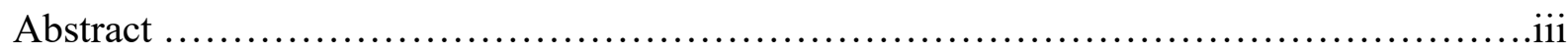

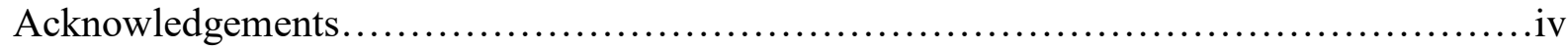

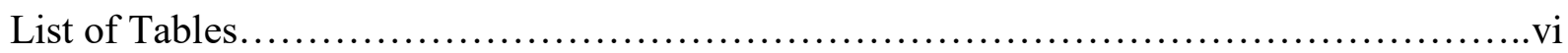

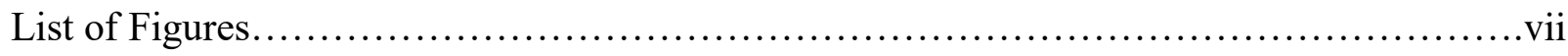

Chapter 1: Overview of the Problem.............................................

Chapter 2: The Multisystemic Stress Response.....................................2

Chapter 3: Maternal Sensitivity and Infant Stress System Development ..................7

Chapter 4: Research Questions and Hypotheses................................... 13

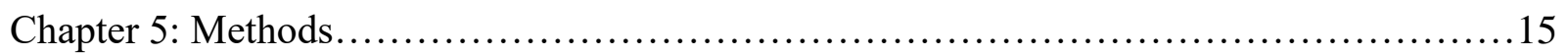

Participants..........................................................

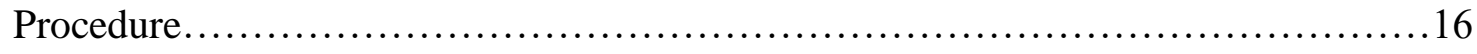

Challenges............................................................. 16

Measures................................................................

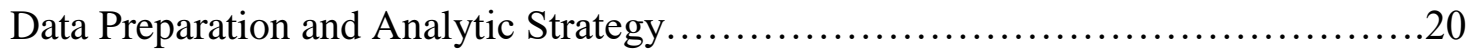

Chapter 6: Results..........................................................24

Preliminary Results........................................................ 24

Main Analyses.......................................................... 24

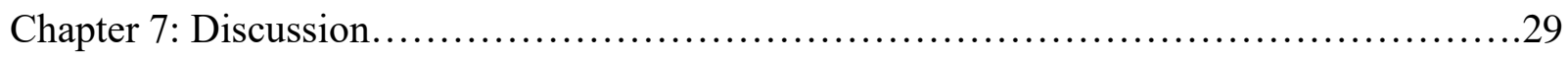

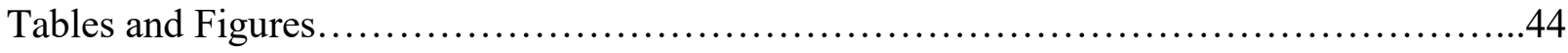

References................................................................52 


\section{LIST OF TABLES}

Table 1. Raw means, standard deviations, medians and interquartile ranges of infant salivary alpha-amylase (sAA) and cortisol concentrations and average maternal sensitivity from the Maternal Behaviour Q-Sort.........................................................44

Table 2. Fixed effect estimates from the 3-level multilevel model predicting log transformed salivary alpha-amylase levels................................................. 45 


\section{LIST OF FIGURES}

Figure 1. Patterns of log transformed sAA and cortisol across stressors (TFP and SSP) and time, at high and low levels of maternal sensitivity ( $\pm 1 \mathrm{SD})$

Figure 2. 2-way interaction between maternal sensitivity and log transformed total cortisol output (i.e., cortisol collapsed across time and stressor) to predict overall levels of log transformed salivary alpha-amylase. Levels of maternal sensitivity represent -1 SD, mean, and +1 SD. Higher levels of maternal sensitivity are associated with greater coordination between

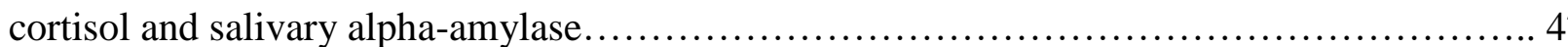

Figure 3. Baseline (5-minutes pre-stressor) coordination of log transformed salivary alphaamylase and cortisol at high and low levels of maternal sensitivity ( $\pm 1 \mathrm{SD})$. Infants of more sensitive mothers had significantly greater baseline sAA and cortisol coordination (i.e., sAA increases as cortisol increases) compared to infants of less sensitive mothers

Figure 4. Peak sampling time coordination of log transformed salivary alpha-amylase and cortisol at high and low levels of maternal sensitivity ( $\pm 1 \mathrm{SD})$. Infants of more sensitive mothers show greater coordination of sAA and cortisol during peak reactivity compared to infants of mothers with lower sensitivity. Peak time represents the +5 minute sample for sAA and the +20 sample for cortisol......................................................................49

Figure 5. Recovery sampling time coordination of log transformed salivary alpha-amylase and cortisol at high and low levels of maternal sensitivity ( $\pm 1 \mathrm{SD})$. Higher levels of salivary alphaamylase are associated with lower levels of cortisol and vice versa, particularly for infants of more sensitive mothers. Recovery represents the +20 minute sample for sAA and the +40 sample for cortisol....................................................................

Figure 6. 2-way interaction between sampling time and sex to predict log salivary alphaamylase, such that female infants had higher levels of sAA on average compared to males, particularly at peak and recovery time points. Note that sampling time is collapsed across stressors........................................................................... 51 


\section{Chapter 1: Overview of the Problem}

The body's ability to adapt to change, termed allostasis, is central to its physical and psychological functioning (McEwen \& Seeman, 1999). An essential allostatic component for maintaining homeostasis is the comprehensive stress response, encompassing integrated processes from the central nervous system, the neuroendocrine system and the immune system (Danese \& McEwen, 2012; McEwen \& Wingfield, 2003). While the systems that constitute the stress response are adaptive in many circumstances, their dysregulation can cause pervasive health consequences (Chrousos, 2009; Danese \& McEwen, 2012; Gunnar \& Quevedo, 2007; McEwen \& Gianaros, 2011; Miller, Chen, \& Zhou, 2007), including increased rates of cardiovascular disease (Black \& Garbut, 2002; Kendler et al., 2009; Steptoe \& Kivimäki, 2012), depression (O’Keane, Frodl \& Dinan, 2012), and impairment of immune system functioning (Epel et al., 2004). Research shows that maladaptive activation (e.g., prolonged or chronic stress) that occurs during developmentally-sensitive periods (e.g., infancy) may be particularly influential in the development of later psychopathology and disease (Gunnar \& Quevedo, 2007; O’Keane et al., 2012; Tarullo \& Gunnar, 2006). Thus, it is of crucial importance to determine what factors positively or negatively influence the stress response during these sensitive periods (McEwen \& Seeman, 1999). Maternal sensitivity, defined as the degree to which a mother responds warmly, appropriately, and contingently to an infant's cues (Ainsworth, Belhar, Waters, \& Wall, 1978), is an early relationship factor found to influence infant stress physiology (e.g., Atkinson et al., 2013; Blair et al., 2008; Feldman, Singer \& Zagoory, 2010). The current study further examines the relationship between maternal sensitivity and infant stress responsivity in a community sample of mother-infant dyads, with a particular focus on sympathetic nervous system (SNS) and hypothalamic-pituitary-adrenocortical (HPA) axis coordination and flexibility. 


\section{Chapter 2: The Multisystem Stress Response}

When facing internal or external threats to homeostasis (i.e., stressors), specific areas in the brain initiate responses from the autonomic nervous system (ANS) and the neuroendocrine system (Ulrich-Lai \& Herman, 2009). The ANS, specifically the sympathetic nervous system (SNS) and the sympathetic-adrenomedullary system, constitute the rapid fight-or-flight response (Gunnar \& Quevedo, 2007; Ulrich-Lai \& Herman, 2009). Together, these branches release two primary catecholamines: norepinephrine and epinephrine. The majority of norepinephrine is synthesized from neurons located within the locus coeruelus of the central nervous system and is released upon sympathetic innervation, although some norepinephrine is also produced in the adrenal glands located above the kidneys. The adrenal glands also synthesize epinephrine, which is released directly into the bloodstream during the fight-or-flight response. Both catecholamines exert effects on targeted cells by binding with noradrenergic and adrenergic receptors. The physiological effects from catecholamines occur rapidly and include increases in metabolic energy (i.e., greater available blood-glucose), heart rate, blood vessel constriction, blood supply to the brain, and muscle constriction (Gunnar \& Quevedo, 2007).

The neuroendocrine system, or HPA-axis, in comparison, produces a slower, longer-acting response via the production of glucocorticoids (Gunnar \& Quevedo, 2007). Once activated, the HPA-axis releases corticotrophin-releasing hormone (CRH) and arginine vasopressin (AVP) from the hypothalamus. These factors then activate the release of adrenocorticotropic hormone (ACTH) from the pituitary gland into the bloodstream, which targets the production and release of cortisol from the adrenal glands. Cortisol is the end produce of the hormonal HPA-axis cascade and produces physiological effects by altering gene expression in targeted cells, particularly those within the brain (de Kloet, 1991). In addition to its role in the stress response, 
cortisol also performs important regulatory functions and is present within the body at all times, following a diurnal course (Lovallo, 2005).

New research suggests that measuring both systems together provides a more accurate representation of the stress response and allows for assessment of system coordination (Ali \& Pruessner, 2012). At the neural level, the SNS and HPA-axis share critical input from the central nervous system, specifically the CRF neurons of the hypothalamus (Engert et al., 2011). However, following centralized innervation, the molecular cascades of these systems are dissociative, producing differing and allied allostatic processes (Sapolsky, Romero, \& Munck, 2000). Evidence from physiological and pharmacological studies show that these systems interact and communicate via inhibitory and excitatory feedback loops (Sapolsky et al., 2000). For example, glucocorticoids can increase the effectiveness of the catecholamines on heart and muscle tissue by decreasing their re-uptake and increasing the sensitivity of $\beta$-adrenergic receptors located within these tissues (e.g., Gibson, 1981; Sakaue \& Hoffman, 1991; for a review see Sapolsky et al., 2000). Further, when pharmacological agents are administered to impede the performance of one of these systems, compensatory responses from the non-targeted system are seen (e.g., Kizildere et al., 2003; Oei et al., 2010; Viru et al., 2007). In addition, the magnitude and trajectory of each system's response differs according to the psychological components of a stressor; specifically, features of uncontrollability or social evaluative threat are known to generate greater HPA reactivity (Dickerson \& Kemeny, 2004), whereas the ANS is sensitive to effort regardless of valence (Chen, Raine, \& Granger, 2015; Frankenhaesuer, 1982; Laurent et al., 2012; Lundberg \& Frankenhaeuser, 1980; Schommer, Hellhammer, \& Kirschbaum, 2003; Ursin, Baade, \& Levine, 1978). Thus, the HPA and ANS likely produce interacting as opposed to summative effects during allostasis (Chen et al., 2015). Delineating the most adaptive patterns in 
multisystem interaction (i.e., coordination) remains an area of ongoing investigation. The available theories and evidence for adaptive coordination will be discussed in detail below.

Non-invasive measurement of these systems has become possible through technological advances over recent decades. Most prominent in the stress literature is the study of cortisol, which can be measured directly and reliability from saliva using immunoassay procedures (Kirschbaum \& Hellhammer, 1994). Acute stressors that feature elements of uncontrollability or social evaluative threat are particularly effective in producing increases in cortisol output, according to meta-analytic findings (Dickerson \& Kemeny, 2004). The direct measurement of norepinephrine and epinephrine, however, requires invasive measurement techniques (i.e., must be measured from blood plasma) (Dvorak, Callés-Escandon, \& Poehlman, 1998). Sympathetic activity can also be measured via heart rate variability (e.g., cardiac systolic pre-ejection period; Newlin \& Levenson, 1979) or skin conductance responses (Lazarus, Speisman, \& Mordkoff, 1963), however, these methods require electrodes connected to wiring and hardware, which can be problematic in studies of young children where mobility is required. For this reason, the majority of developmental stress research (and stress research generally) has focused solely on understanding how different factors influence the HPA-axis. The investigation of a singlesystem, however, neglects the comprehensive, multi-system nature of the stress response.

Fortunately, cortisol is no longer the only stress biomarker that can be reliably measured in saliva. Salivary alpha-amylase (sAA) has gained recognition as a reliable indicator of ANS activity. Salivary alpha-amylase is most often cited as a measure of sympathetic activity (Granger, Kivlighan, El-Sheikh, Gordis, \& Stroud, 2007), though there is some evidence that small amounts are secreted by parasympathetic innervation (Bosch, Veerman, de Geus, \& Proctor, 2011; Nater et al., 2006). Alpha-amylase is an enzyme produced in the salivary glands, 
primarily the parotid gland, that aids in digestion (Nater \& Rohleder, 2009) and provides immunological protection against certain bacteria (Scannapieco, Torres, \& Levine, 1993). Salivary alpha-amylase is not produced in newborn infants but rapidly increases in concentration around nine months, reaching adult levels between 3 and 6 years of age (O’Donnell \& Miller, 1980). Salivary alpha-amylase is found to increase in response to physiological and psychological stressors in adults (Chatterton, Vogelsong, \& Hudgens, 1996; Nater et al., 2005; Nater et al., 2006; Rohleder, Nater, Wolf, Ehlert, \& Kirshbaum, 2004; Skoluda et al., 2015), infants (Laurent, Ablow, \& Measelle, 2012), toddlers (Fortunato et al., 2008), preschoolers (Spinrad et al., 2009), and adolescents (Stroud et al., 2006). Thus, sAA has aided in expanding the developmental stress literature to include the other systems that are integral to a comprehensive and adaptive stress response.

Two components of an adaptive stress response are coordination and flexibility (Atkinson et al., in press). As mentioned above, the multiple stress systems work in combination to overcome allostatic demands. Coordination refers to the relationship between the different stress system responses, i.e., the degree to which the SNS and the HPA-axis respond to stress in a coordinated (or synchronous) fashion. Synchrony is not to be confused with uniform responding or redundancy but rather the interactive influence of one system on another, i.e., the magnitude and direction of responding from one system is related to the magnitude and direction of the other (Chen et al., 2015). Further, this interaction may be influenced by additional environmental or genetic moderators, such as those at play during early development. The exact pattern of adaptive coordination and its interplay with developmental moderators is under contention and will be discussed below. Flexibility is the ability to produce an appropriate, context-specific stress response. A flexible stress response matches situational demands as necessary, in 
comparison to blunted or superfluous responses. Flexibility is not merely variability in stress responding. As illustrated by Atkinson and colleagues (in press), an individual may produce variable SNS or HPA reactivity in response to different stressors but the pattern of variability must match the intensity and specificity of the stressor to be considered flexible. For example, an individual who produces a robust HPA response to a frustrating task but fails to mount a response to a socially-threatening environment is demonstrating variability but not flexibility in their stress responding. Developmental factors, both environmental and physiological/genetic, are expected to explain individual variations in flexibility. 


\section{Chapter 3: Maternal Sensitivity and Infant Stress System Development}

The quality of early attachment relationships has been found to influence the development of stress physiology (Haley \& Stansbury, 2003; Nachmias et al., 1996). In their seminal work, Meaney and Szyf (2005) demonstrated the importance of maternal care on stress system development in rat models. Greater maternal care (e.g., the frequency of licking and grooming behaviours) resulted in epigenetic changes that produced favourable and persistent alterations in rat pup stress physiology (i.e., variation in the expression of glucocorticoid receptors). Though causal examinations are considerably more difficult with human subjects, multiple studies have found significant associations between maternal sensitivity and infant HPA function (e.g., Atkinson et al., 2013; Hibel, Granger, Blair, Finegood, The Family Life Project Key Investigators, 2014; Laurent et al., 2012; Letourneau, Watson, Duffett-Leger, Hegadoren, \& Tryphonopoulos, 2011); higher levels of maternal sensitivity have been correlated with lower baseline cortisol concentrations, greater reactivity during the course of a stressor and less time for baseline recovery in infants (Blair, Granger, Willoughby, \& Kivlighan, 2006; Blair et al., 2008; Spanglar, Schieche, Ilg, Maier, \& Ackermann, 1994). Other studies have found contrasting evidence (e.g., Jansen, Biejers, Riksen-Walraven, \& de Weerth, 2010; Thompson \& Trevathan, 2009), which may relate to methodological differences or the influence of moderators (Atkinson et al., 2013). Regardless, there is building evidence for the important relationship between early maternal care and infant HPA-axis development.

Considerably less research has examined the influence of maternal sensitivity on infant SNS system functioning and the interconnectivity, or coordination, of the SNS and HPA systems (Andrews et al., 2013). To address this research gap, there has been a call for studies that assess the interplay of the stress systems to better understand how these systems work in collaboration 
to achieve allostasis (Ali \& Pruessner, 2012; Andrews et al., 2013; Bauer, Quas, \& Boyce, 2002; Granger et al., 2006). A few theories have been put forward to explain system coordination and how it relates to developmental and contextual factors.

Among the first of these theories was that of Bauer and colleagues (2002) who proposed that “allostatic load may manifest as dysregulated patterns of stress response across systems rather than alterations in individual system activity" (pg. 104). In their paper, they proposed two contrasting hypotheses: 1) an "additive" model, where risk is related to over-activation of both systems (i.e., mid-level arousal or high-with-low arousal in both systems is optimal), or 2) an “interactive model", where asymmetry between stress systems (i.e., low reactivity in one system with high reactivity in another) is predictive of risk. Evidence has thus far been equivocal, with results available in support of each model. Corroborating the additive model, greater self- and parent-reported aggression in adolescents was related to low cortisol and sAA reactivity in response to a social stressor, but not in adolescents with low cortisol and higher sAA reactivity (Gordis, Granger, Susman, \& Trickett, 2006). Further, higher levels of both basal cortisol and sAA were associated with increased internalizing and externalizing behaviours in 8 and 9-yearolds (El-Sheikh, Erath, Buckhalt, Granger, \& Mize, 2008). Yet, in support of the interactive model, no associations between sAA and cortisol reactivity were found in a 9 to 14-year-olds with a history of maltreatment, whereas a comparison group without history of maltreatment demonstrated symmetry between sAA and cortisol (Gordis, Granger, Susman, \& Trickett, 2008). Moreover, in adolescents (aged 12 to 19) displaced by Hurricane Katrina, asymmetry between basal sAA and cortisol was related to mood problems, whereas symmetry (e.g., high sAA and cortisol) was not (Vigil, Geary, Granger \& Flinn, 2010). 
In extension to Bauer et al.'s hypotheses, Andrews and colleagues (2013) proposed the stress coherence/compensation model, which suggests that stress system asymmetry is the product of one system becoming dysregulated and the other system compensating for it. For example, if one system (e.g., the HPA-axis) were to become blunted (e.g., via chronic stress as in Miller et al., 2007), the stress coherence/compensation model predicts that the SNS would compensate by generating an overactive response (Andrews et al.). The stress coherence/compensation model also argues that psychological measures of stress (e.g., stress perception according to a visual analogue scale) constitute an important third system requiring measurement.

Furthermore, Del Guidice and colleagues (2011) put forward the adaptive calibration model, which suggests that children's stress system trajectories adapt to specific developmental and environmental demands. Del Guidice et al. propose four trajectories and their predicted environmental correlates: 1) the sensitive pattern, indicated by high HPA and medium-high SNS reactivity, related to sensitive parenting, 2) the buffered pattern, characterized by moderate reactivity in both systems, related to over-stimulation during development, 3) the vigilant pattern, with high reactivity in both systems but lower reactivity in the parasympathetic nervous system (indicating difficulties with relaxation) with differential gender-based differences in behavioural outcomes, and 4) the unemotional pattern, with low reactivity overall, theoretically linked to neglect, trauma and/or chronic stress (Del Guidice et al.). The adaptive calibration model extends allostatic load theories by incorporating the importance of life history into how allostatic load shapes stress system development and, subsequently, disease processes (Ellis \& Del Guidice, 2014). The quality of early attachments is consistent with life history events that the adaptive calibration model seeks to address. From an evolutionary perspective, the adaptive 
calibration model also suggests that none of the trajectory patterns are fundamentally 'dysfunctional' but rather adaptive in different contexts (Ellis \& Del Guidice). This is similar to research supporting differential susceptibility (gene $\mathrm{x}$ environment interactions) in the context of early care (Belsky \& Pleuss, 2009), as has been found within our lab on dopamine-related genes and cortisol reactivity in infants of mothers with depressive symptoms (Ludmer et al., 2015). Further research incorporating multiple stress system measures is necessary to test the adaptive calibration model in developmental contexts.

In addition to coordination, the quality of early care may also influence infant stress system flexibility, as indicated by appropriate stress reactivity to the potency and duration of a stressor (McEwen \& Seeman, 1999). The tendency for some stressors to elicit a greater response of a particular stress system compared to others is referred to as stressor specificity (Gunnar, Talge \& Herrera, 2009). Systematic reviews of experimental HPA-axis activation find that certain laboratory stressor paradigms are more potent stressors than others (Dickerson \& Kemeny, 2004; Jansen et al., 2010; Gunnar et al., 2009); a meta-analysis currently under way in our lab finds similar sAA patterns for stressor paradigms, at least in adult populations (Villani \& Jamieson, in prep). Despite these findings, there exists a paucity in the developmental stress flexibility research, particularly the flexibility of infant SNS reactivity, and how this relates to contextual variables (e.g., maternal care). In particular, there is limited research on infant intra-individual stress flexibility (i.e., differential stress responses across stressors within individuals). Our lab recently found evidence for intra-individual infant HPA-axis flexibility across two different challenges, such that infants produced greater cortisol outputs when exposed to a parental separation procedure compared to a toy frustration procedure (Atkinson et al., 2013). Importantly, the degree of intra-individual flexibility was related to maternal sensitivity, such 
that infants of more sensitive mothers displayed greater cortisol variability across the two challenges compared to infants of less sensitive mothers (Atkinson et al.). In a study of 18month-old infants, Laurent and colleagues (2012) also demonstrated variability in infant cortisol and sAA trajectories across different stressors. They found that cortisol reactivity was higher following the strange situation procedure, whereas sAA trajectories were higher following a clean-up and emotion-induction task. Mothers and infants both demonstrated the same patterns of reactivity in response to the stressors suggesting shared attunement in their physiological relationship. This study, however, did not directly assess stress system flexibility per se, though they did find some evidence linking behavioural factors (i.e., temperament) to distinct patterns of cortisol and sAA responding. For example, they found that infants with greater surgency (as indicated by maternal reporting on the Early Childhood Behavior Questionnaire) had higher cortisol in the strange situation procedure. Another sample of 6-month-old infants and mothers from low-income families found evidence for parenting behaviours (measured via self-report) on sAA trajectories following novel and frustrating stressor tasks (Kivlighan, 2006). Mothers who demonstrated high levels of detached parenting had infants with low or non-existent sAA reactivity in response to the challenges. Anxious mothers were found to have the opposite pattern, with infants producing greater sAA reactivity compared to low anxious mothers. An untested explanation for these reversed findings may be that anxious mothers signal to their infant that the situations is of heightened threat or demand, whereas a detached parent may not give their child these cues. Kivlighan's dissertation findings are the first (to my knowledge) to demonstrate a relationship between aspects of maternal care and infant SNS responsivity.

In sum, important components of stress physiology are shaped in early childhood, in part by the quality of early care. However, few studies have concurrently studied the relationship 
between the SNS and the HPA-axis in the context of maternal sensitivity. In particular, there is a lack of research examining the relationship between maternal sensitivity and infant SNS system flexibility, the coordination of HPA and SNS systems, and coordination of these systems in the context of intra-individual flexibility. The current study addresses these gaps in the literature. 


\section{Chapter 4: Research Questions and Hypotheses}

This investigation furthers the developmental stress literature by examining the relation between maternal sensitivity and the coordination between infant sAA and cortisol reactivity, and infant sAA flexibility across two stressor challenges (intra-individual stress flexibility). Specifically, the following research questions were explored:

1) Is maternal sensitivity related to the interconnectivity of infant stress systems, specifically the HPA-axis and the SNS?

Hypothesis 1: Maternal sensitivity moderates the relationship between infant sAA and cortisol, such that higher levels of maternal sensitivity relate to greater coordination (i.e., symmetry) between infant sAA and cortisol response trajectories.

Previously described models of coordination suggest that interconnectivity between stress systems is an adaptive component of stress physiology that may be fostered by the quality of

early care (Bauer et al., 2006; Andrews et al., 2013; Del Guidice et al., 2011). Infants with higher levels of maternal sensitivity are expected to produce sAA and cortisol trajectories that are similar to the sensitive pattern described by Del Guidice and colleagues (2011), specifically with infants raised in more sensitive environments demonstrating greater sAA and cortisol basal activity and responsivity to acute stress.

2) Is maternal sensitivity related to infant sAA flexibility across different stressors?

Hypothesis 2: Higher levels of maternal sensitivity relate to greater infant sAA response flexibility (i.e., appropriate variability) across stressors.

Atkinson et al. (2013) found that infants of more sensitive mothers had greater intraindividual cortisol flexibility across different stressors. In line with this finding, it is expected that a similar pattern will exist in sAA responding, with infants of more sensitive mothers 
generating greater flexibility in their SNS response across differentially challenging stressors. However, as the SNS is more often associated with effortful or frustrating tasks (Frankenhaesuer, 1982; Laurent et al., 2012; Lundberg \& Frankenhaeuser, 1980; Ursin, Baade, \& Levine, 1978), it was predicted that infants demonstrate greater sAA reactivity in response to a toy frustration procedure as opposed the strange situation procedure, which elicits more relational threat.

3) Is maternal sensitivity related to the interaction between stress system coordination and flexibility?

Hypothesis 3: The degree of coordination between infant sAA and cortisol differs across two different challenges (i.e., flexibility), and this is related to the degree of maternal sensitivity. The expected direction of this effect is unknown as it has not been assessed in previous studies. As such, this is an exploratory hypothesis. 


\section{Chapter 5: Methods}

\section{Participants}

Following approval from the Research Ethics Boards at the Centre for Addiction and Mental Health and Ryerson University, a low-risk community sample of 297 mother-infant dyads were recruited from in-person visits and flyers posted at community centres, activity centres and infant-related events to participate in our laboratory's original longitudinal study (Atkinson et al., 2013; Khoury et al., 2016; Ludmer et al., 2015; Pereira et al., 2012). Dyads were eligible for participation if mothers spoke fluent English, were over 18 at the time of their child's birth, had no major medical conditions, and if the infant was developing typically and had an uneventful childbirth. Saliva samples timed for sAA collection did not begin until late in the study (as the role of sAA as a reliable biomarker became more established), such that 117 dyads were not assessed at all. From the remaining dyads, the current study assayed a subsample $(\mathrm{N}=$ 125) selected for completeness of data (i.e., saliva samples available for both challenges; 55 infants with incomplete sAA data were not included due to funding constraints); this sample did not significantly differ from the original sample on any important aspect (e.g., sex, income, cortisol reactivity). Infants (52\% female) were between 14 and 18 months old at the initial home visit $(M=15.17, S D=.84)$ and between 14 and 19 months at the laboratory visit $(M=16.46, S D$ $=.90)$. Maternal age at the 15 -month home visit ranged from 22 to 46 years $(M=33.27, S D=$ 4.54). The majority of mothers were in a relationship (95.2\%) and had a median income of $\$ 35,001$ to $\$ 70,000\left(25^{\text {th }}\right.$ and $75^{\text {th }}$ percentiles were less than $\$ 20,000$ and $\$ 35,001-\$ 70,000$, respectively). The median reported income for spouses was $\$ 35,001$ to $\$ 70,000\left(25^{\text {th }}\right.$ and $75^{\text {th }}$ percentiles were $\$ 35,001-\$ 70,000$ and $\$ 70,001-\$ 92,000)$. Mother's highest level of education was self-reported as primary (1.6\%), secondary (5.6\%), community college (26.4\%), university 
(40.8\%), or post-graduate degree/diploma (25.6\%). Six mothers (4.8\%) were smokers and had an average of 4.25 cigarettes daily. Ancestry was self-reported as Caucasian $(n=97,78.2 \%)$, Asian $(\mathrm{n}=9,7.3 \%)$, African American $(\mathrm{n}=2,1.6 \%)$, and other (including Hispanic, East Indian, Middle Eastern, South Asian and Mixed; $\mathrm{n}=16,12.9 \%$ ). One mother did not report ancestry. Procedure

Dyads participated in two study sessions. A home visit was conducted at age 15 months and involved a two-hour period of naturalistic observation, completion of maternal questionnaires and participation in a toy frustration procedure (TFP). One month later (age 16 months), dyads participated in a strange situation procedure (SSP) during a laboratory visit. (The SSPs were utilised as an infant stressor and were not coded for attachment classifications.) During both the TFP and the SSP, saliva samples were collected from the mother and infant at four time points (baseline, and 5 minutes, 20 minutes and 40-minutes post-stressor). All visits were scheduled in the morning (between 8 and $11 \mathrm{am}$ ) to account for infant diurnal patterns of cortisol and to reduce the likelihood of disrupting the diurnal course due to interrupting regularly scheduled naps or feeding times (Goldberg et al., 2003; Gunnar \& White, 2001; van Bakel \& Risken-Walraven, 2004).

\section{Challenges}

Toy Frustration Procedure. The TFP (Braungart-Rieker \& Stifter, 1996) is a stressor paradigm designed to induce frustration in infants and toddlers. Mothers were instructed to amuse their infant with an appealing toy (provided by the research assistant) for 90 seconds, followed by a 90-second interval during which the mother concealed the toy in a clear plastic container out of the child's reach. These two steps were immediately repeated, for a total of four 90-second intervals. In both concealment steps, infants would attempt to retrieve the toy, though 
this was most difficult in the last interval where the container was sealed. Intervals were terminated if infants cried steadily for more than 20 seconds $(8 \%$ of sessions were terminated early, though termination status was missing for an additional $32 \%$ of cases).

Strange Situation Procedure. The SSP (Ainsworth et al., 1978) is a separation-reunion paradigm originally designed to assess mother-infant attachment styles that also functions as a potent relational stressor. The SSP involves eight three-minute intervals, two of which involve mother-infant separations (while the mother watches from an observation room). Separation intervals were terminated if the infant cried steadily for more than 20 seconds (36\% of SSPs had at least one interval terminated due to crying).

The TFP and SSP are differentially effective in evoking a cortisol response, rendering them an ideal pair for assessing HPA-axis intra-individual variability in physiological reactivity (Atkinson et al., 2013). The SNS is also known to produce differential reactivity depending on the type of stressor, typically with greater reactivity in response to effortful challenges (Frankenhaesuer, 1982; Laurent et al., 2012; Lundberg \& Frankenhaeuser, 1980; Ursin, Baade, \& Levine, 1978). However, effort was likely a component of both the TFP and the SSP. For example, in the TFP, infants would have produced effort in attempt to retrieve the toy (particularly in the second concealment when the container was locked), but many infants also tried to leave the room during the SSP to find their mother (a behaviour that may also be indicative of effort).

\section{Measures}

Maternal Sensitivity. The Maternal Behaviour Q-Sort (MBQS; Pederson et al., 1990) is a measure of maternal sensitivity that yields robust effect sizes in the context of attachment security ( $r=.48$; Atkinson et al., 2000) and cortisol secretion (Atkinson et al., 2013). Two 
trained female research assistants observed dyads in their homes over the course of two hours, which included the time period where mothers were instructed to fill out questionnaires with infant present; this serves to divide mothers' attention and may augment the relation between maternal sensitivity and infant behaviour (Atkinson et al., 2000; Pederson et al., 1990). The two observers sorted cards describing maternal behaviour (e.g., "waits for baby's response in interactions") into piles according to how well they describe the mother in question. The observed sorts were then compared to the sort of a "prototypically sensitive" mother and the correlation serves as a measure of sensitivity (Pederson et al., 1990). In this study, the average value of both observers was used. An inter-rater reliability (intra-class correlation) of .88 was obtained.

Saliva Collection and Assay. Mothers and infants were instructed to refrain from eating or drinking anything but water, or brushing their teeth in the hour leading up to each visit to avoid sample contamination (Kirschbaum \& Hellhammer, 1994; Mackie \& Pangborn, 1990). Due to the timing of the visits, compliance with these instructions was poor: prior to the home visit, $42.4 \%$ of infants ate or drank something other than water, as did $31.2 \%$ infants prior to the lab visit.

Whole saliva samples were collected using Sorbettes (Salimetrics, State College, PA) that were placed in between the gum and the cheek and held there for 60 seconds. During each challenge, four samples were collected at baseline (5-minutes pre-stressor) and 5, 20, and 40 minutes post-stressor from both mother and infant. Saturated Sorbettes were deposited directly in 2-mL cryovials and stored at $-70^{\circ} \mathrm{C}$ in an ultra-low temperature freezer.

For assay, only three of the four samples were used for either sAA or cortisol to account for the differential time course of the SNS and HPA-axis; for sAA, baseline, 5, and 20 minute 
samples were used, whereas baseline, 20, and 40 minute samples were used for cortisol. These samples were matched according to their respective baseline, peak, and recovery stages to account for the time-lag between systems, which Laurent and colleagues (2013) titled 'matched phase coordination' and recommended as a superior method for calculating multisystem coordination.

Cortisol samples were previously assayed for both mother and infant using a high sensitivity enzyme immunoassay kit (Salimetrics, State College, PA). For cortisol assay, samples were thawed and centrifuged for 10 minutes at $3000 \mathrm{rpm}$ at $4^{\circ} \mathrm{C}$. All samples from an individual were conducted on the same day. Each sample was done in duplicate and averaged values were used in subsequent analysis. Following assay, cryovials with remaining saliva were refrozen at $70^{\circ} \mathrm{C}$. Inter-assay variance was $10.6 \%$ and inter-assay variance was $8.3 \%$ for low value samples and $6.9 \%$ for high value samples.

For sAA assay, infant samples were re-thawed and centrifuged for 15 minutes at 3000 rpm. All assays were conducted using a high sensitivity immunoassay kit (Salimetrics, State College, PA) and a programmable microplate reader (BioTek, Winooski, VT) preheated to $37^{\circ} \mathrm{C}$ and set to a $405 \mathrm{~nm}$ filter. All samples for each infant were conducted on the same day. Duplicates of each sample were assayed and average values are reported. Inter-assay variance was $14.14 \%$ and intra-assay variance was below $4 \%$ for low, medium and high samples.

Potential Covariates. Known covariates of cortisol and sAA were considered in the experimental design and were accounted for, as necessary, in the statistical analyses. As described, mothers were instructed to refrain from drinking and eating for one hour prior to the study visits. However, given the low compliance rate, time of last meal/drink were included as covariates. Further, certain demographic factors have been found to influence these biomarkers; 
socioeconomic status has been linked to child cortisol response (Bauer et al., 2002), and sex of infant has received mix-evidence on its relationship to sAA reactivity (Frankenhaeuser, 1982; Takai et al., 2007; Vigil et al., 2010). In the present study, socioeconomic status was captured via maternal self-report on maternal education, household salary and marital status (married/cohabiting compared to single). These potential covariates were assessed against cortisol and sAA secretion and only covariates found to significantly influence the model were included.

\section{Data Preparation and Analytic Approach}

Data were assessed visually and statistically for skew and outliers. Log transformations were used to reduce the positive skew of cortisol and sAA values. Missing data were accounted for via multiple imputation procedures (Little \& Rubin, 1987; Schafer \& Olsen, 1998). Extreme outliers (values \pm 3.29 standard deviations) were contributed from a single infant who produced salivary alpha-amylase values below natural limits. As such, this infant's data was dropped from the subsequent analyses. An additional 12 infants were excluded from the analyses due to difficulties obtaining saliva samples in the required time range for salivary alpha amylase. Though our research assistants were well-trained and experienced, infant fussiness or prolonged irritation following the stressors led to cases of undesirable variability in salivary sampling (a phenomenon we suspect is not specific to our lab). In an effort to conserve power and reliability in interpreting peak and recovery periods of sAA, peak sampling times occurring outside of 4-10 minutes were excluded ( $M=5.61, S D=.19)$, as were recovery samples outside of 15-30 minutes $(M=20.64, S D=.19$; final $\mathrm{N}=112)$.

Data were analyzed using multilevel modelling (MLM) with maximum likelihood (Raudenbush, Bryk, Cheong, Congdon, \& du Toit, 2011) to account for interdependence of physiological measures within individuals. Interclass correlations depicting the variance 
accounted for between sample time and challenge $(\mathrm{ICC}=.53)$ and individual $(\mathrm{ICC}=.79)$

corroborate the use of nesting procedures. A three-level model was used to assess infant sAA and cortisol trajectories across time (Level 1), and challenge (Level 2), nested within individual infants (Level 3). As opposed to traditional forms of linear regression, examination of trajectories using MLM provides information about differential rates of change across and within individuals (growth curves, i.e., slopes) and idiosyncratic initial status along the dependent variable (i.e., intercepts), and how selected predictors influence these factors (Hruschka, Kohrt, \& Wrothman, 2005; Singer \& Willet, 2003; Willett \& Sayer, 1994). Similarly, trajectories provide greater information than computation of area under the curve calculations or other composite forms of measurement that result in the loss of important intra-individual variation in physiological data (Hruschka et al.). Additionally, MLM has the added benefit that uniform time measurements are not necessary (Garson, 2013; Hruschka et al.).

To test the multiple hypotheses put forward, the full model of interest was inputted into multilevel modeling software (HLM-7, Scientific Software International): sAA was selected as the outcome variable (as opposed to cortisol which was the focus of previous work on this sample; Atkinson et al., 2013); cortisol (group mean centered) and time (coded as baseline $=0$, peak $=1$ and recovery $=2 ;$ uncentered) were entered as level-1 predictors; stressor challenge (uncentered, dummy-coded as toy frustration $=0$, strange situation $=1$ ) as a level-2 predictor; and at the individual level (level-3), maternal sensitivity was grand mean centered. According to guidelines on model evaluation (Cohen, Cohen, West, \& Aiken, 2013; McCoach \& Black, 2008), non-significant interactions of the highest level (beginning with the four-way interaction) were systematically removed from the model to create the most parsimonious fit. The end product of this process included a three-way interaction between maternal sensitivity, sample time and 
cortisol, as well as additional two-way interactions and significant main effects. The influence of potential covariates was then tested on the parsimonious model. Feeding time and wake time were entered as level-2 factors and sociodemographic factors and infant sex were entered as level-3 predictors. Only infant sex was found to significantly influence the model and was thus retained as a level-3 predictor. Infant sex also produced a significant two-way interaction with time. Sample time, cortisol, challenge, sex and the hypothesized moderator maternal sensitivity were included as fixed effects and interpreted using final estimation with robust standard errors. Random effects (slopes) for time, cortisol and stressor were not significant and were therefore not included in the final model. Random intercepts and residual variances were included. The final model is outlined by equation 1 . Level-1 residuals did not violate the assumption of homogeneity of level-1 errors, nor did ordinary least squares (OLS) residuals for level-2.

Significant interactions were further analysed using an online multilevel model interaction computation tool (Preacher, Curran, \& Bauer, 2006). This tool plots the regressed slopes of predictors on the outcome variable for either two-way or three-way interactions. It uses covariance and variance input from the final multilevel model and requires the selection of conditional values for each predictor of interest. Where applicable, these conditional values were selected as the mean and \pm 1 standard deviation values for cortisol and maternal sensitivity, or for sampling time, as the integer representing either baseline (0), peak (1), or recovery (2). This computation tool also produces simple intercept and simple slopes analyses that compare regressed slopes to a slope of zero (i.e., assessing the traditional null hypothesis). This study's hypotheses, however, were primarily concerned with comparisons between slopes (e.g., comparison of slopes between high and low levels of maternal sensitivity), which are supported 
by the presence of significant interactions (Aiken \& West, 1991). Thus, simple slopes analyses are only included where relevant.

$$
\text { sAA }=\text { Y00 + Y001*Maternal Sensitivity }+ \text { Y002*Sex }+ \text { Y010*Stressor }+
$$

Y100*Cortisol + Y101*Cortisol*Maternal Sensitivity + Y200*Time + Y201*Time*Maternal Sensitivity + Y202*Time*Sex + Y300*Time*Cortisol + Y301*Time*Cortisol*Maternal

$$
\text { Sensitivity }+\mathrm{r} 0+\mathrm{u} 00+\varepsilon
$$




\section{Chapter 6: Results}

\section{Preliminary Results}

Infant sAA was not significantly affected by sociodemographic factors (maternal education, household income, or marital status), wake or feeding time, or maternal smoking status. The influence of these covariates on cortisol was also tested (e.g., the interaction between cortisol values and wake/feeding time on sAA) but these interactions were non-significant. Infant sex was found to have a main effect on infant sAA $(\beta=.11, S E=.05 t(109)=2.71, p=.027)$, such that female infants had higher average concentrations of sAA than males. Table 1 displays the means, standard deviations, medians and interquartile ranges for untransformed sAA, cortisol, and maternal sensitivity. Patterns of sAA and cortisol across visit and time at high and low levels of maternal sensitivity (one standard deviation above and below the mean) are depicted visually in Figure 1.

\section{Main Analyses}

\section{Full Model}

The final parsimonious model (see equation 1) fit the data significantly better than the null model $\left(x^{2}(10)=43.11, p<.001\right)$. Parameter coefficients and standard errors of the model's fixed effect factors, calculated using log transformed sAA and cortisol values, are depicted in Table 2. In addition to the significant main effect of sex, there was a significant main effect of stressor $(\beta=.10, S E=.09, t(111)=4.99, p<.001)$, such that infant's overall sAA trajectories were greater during the strange situation compared to the toy frustration procedure. There was a significant three-way interaction between sampling time, cortisol and maternal sensitivity $(\beta=-$ $.17, S E=.07, t(329)=-2.36, p=.019)$. Further, there was a significant two-way interaction between cortisol and maternal sensitivity $(\beta=.29, S E=.11, t(329)=2.55, p=.011)$ and a 
marginally significant two-way interaction between time and maternal sensitivity $(\beta=.08, S E=$ $.04, t(329)=1.84, p=.067)$. In addition, there was a significant two-way interaction between sample time and $\operatorname{sex}(\beta=.02, S E=.01, t(329)=2.07, p=.040)$. No other interactions or main effects were statistically significant.

The model also contained significant random effects. The level-1 intercept had significant variability $\left(\right.$ variance $\left.=.013, s d=.11, x^{2}(111)=407.25, p<.001\right)$, indicating that there were significant individual differences between infant levels of sAA. Thus, the level-1 intercept was allowed to vary randomly to improve the model's fit. Further, there was significant variability in the level-2 intercept component $\left(\right.$ variance $\left.=.058, s d=.24, x^{2}(109)=827.35, p<.001\right)$, indicating a significant stressor effect on infant levels of sAA. This corroborates the significant main effect of stressor mentioned above and also supports the use of nesting procedures as indicated by the levels of the ICC (Garson, 2013). There was no significant benefit for allowing random slope components in the model, contrary to expectations that there would be idiosyncratic variability in slope (i.e., trajectories) across time and stressor.

\section{Hypothesis 1: Maternal sensitivity as a moderator of the relation between infant}

\section{SAA and cortisol basal activity and reactivity}

We hypothesized that maternal sensitivity moderates the coordination between infant sAA and cortisol basal activity and responsivity to acute stress, such that higher levels of maternal sensitivity would relate to greater coordination between sAA and cortisol response trajectories. The significant two-way interaction between cortisol and maternal sensitivity supports maternal sensitivity as a moderator of the relationship between cortisol and salivary alpha-amylase. Figure 2 portrays the positive linear relationship between cortisol and sAA at low, average and high levels of maternal sensitivity. The regression of cortisol on sAA was 
significantly different from zero at average $(\beta=.13, S E=.064, z=1.97, p=.049)$ and high levels of maternal sensitivity ( $\beta=.21, S E=.094, z=2.25, p=.025)$, but not at low levels ( $\beta=$ $.04, S E=.040, z=1.05, p=.29$ ). Specifically, at higher levels of maternal sensitivity, sAA increases as cortisol increases. The influence of maternal sensitivity on sAA/cortisol coordination, however, must be assessed in the context of the significant three-way interaction between cortisol, maternal sensitivity and time.

The significant three-way interaction between cortisol, maternal sensitivity and time indicates that the regression of cortisol on salivary-alpha amylase varies across levels of maternal sensitivity and sampling time. At baseline (Figure 3), sAA increases positively with increases in cortisol at high levels of maternal sensitivity but not at lower levels of maternal sensitivity. In other words, infants with more sensitive mothers had greater basal sAA and cortisol synchrony. At peak sampling time (Figure 4), this pattern persists, though to a lesser degree than at baseline. During recovery (Figure 5), sAA and cortisol are negatively related, such that higher levels of sAA are related to lower levels of cortisol and vice versa; this is true for both high and low levels of maternal sensitivity but to a greater degree for infants of more sensitive mothers.

\section{Hypothesis 2: Higher levels of maternal sensitivity relate to greater infant sAA} response flexibility across stressors

We predicted that infants of more sensitive mothers would have greater sAA betweenchallenge variability, i.e., greater flexibility in their sAA response. There was a significant main effect of stressor, providing evidence for sAA output variability across challenges for infants on average, such that sAA values were higher following the SSP than the TFP. This was contrary to initial predictions that sAA output would be higher in response to the TFP. No significant interactions were found between stressor and additional variables (e.g., maternal sensitivity) as 
hypothesized, though visual inspection of Figure 1 demonstrates distinctive patterns of sAA reactivity across stressors for low and high levels of maternal sensitivity. Infants of more sensitive mothers appear to mount an appropriate sAA response for both stressors, but infants of less sensitive mothers fail to mount a peak sAA response in both stressors. In addition to a failed peak response, vulnerable infants (i.e., those with less sensitive mothers) produced higher sAA output during the unstructured recovery period instead of the structured stressor. The significant three-way interaction between cortisol, maternal sensitivity, and time described above coupled with the significant main effect of stressor highlights trends in infant flexibility.

\section{Hypothesis 3: The degree of coordination between infant sAA and cortisol differs} across challenge (i.e., flexibility), and is related to the degree of maternal sensitivity

We explored whether coordination between infant sAA and cortisol would be contextspecific, i.e., whether there would be different patterns of coordination across stressors (coordination $\mathrm{x}$ flexibility interaction). As mentioned, there was no significant interaction between stressor and maternal sensitivity. However, nuances in the contextual, stressor-specific patterns of coordination can be seen visually in Figure 1, which plots sAA/cortisol trajectories as a function of low (blue lines) and high (red lines) maternal sensitivity for both stressors (TFP and $\mathrm{SSP}$ ). In both the TFP and the SSP, it is clear that infants of more sensitive mothers produce sAA/cortisol trajectories that are more similar than their low sensitive counterparts. In the TFP, infants with more sensitive mothers produce increases in sAA as they produce decreases in cortisol. For infants of less sensitive mothers, this expected pattern of coordination did not occur. In the SSP, infants of more sensitive mothers produce complementary increases in sAA and cortisol that decrease over recovery. In comparison, sAA and cortisol are asynchronous for low sensitivity infants in the SSP, where neither system produces the expected peak response. 


\section{Influence of Sex}

Infant's biological sex was found to significantly interact with sampling time. Figure 6 depicts mean levels of sAA for each gender by each time point (collapsed across stressor and levels of maternal sensitivity). In line with the significant main effect of sex, females produce significantly more sAA across all sampling time points (baseline, peak, and recovery). The simple slope of male sAA across time was not significantly different from zero $(\beta=.0046, S E=$ $.013, z=.34, p=.73)$, indicating that males, on average, produce similar levels of sAA across each time point. In comparison, female infants produced increasing levels of sAA across time ( $\beta$ $=.026 S E=.012, z=2.13, p=.033)$, with the highest average values found at recovery, followed by peak. In sum, female infants, regardless of environmental demands, maternal sensitivity or cortisol reactivity, appear to have a more reactive SNS response and a slower SNS recovery rate than their male counterparts. 


\section{Chapter 7: Discussion}

This study examined the relationship between maternal sensitivity and two adaptive components of infant stress physiology: 1) coordination between basal activity and reactivity of the SNS and HPA-axis, and 2) flexibility of the SNS in response to two differentially challenging acute stressors. Previous work has assessed maternal sensitivity and related parenting constructs in the context of a single stress system (i.e., the HPA-axis), which neglects the underlying integration of multiple systems comprising the comprehensive stress response. Of the available multi-system studies, coordination and flexibility have been assessed in relation to alternative variables (e.g., infant temperament, maternal anxiety) but this study is the first to concurrently examine multiple systems in the context of maternal care using a robust observational measure of sensitivity. This study also adds the novel contribution of assessing the interaction between coordination and flexibility within individual infants (i.e., exploring the possibility that the construct of flexibility includes differential patterns of coordination between stressors of varying potency and specificity). Further, this study assessed the coordination, flexibility, and interaction hypotheses using a powerful statistical approach that accounted for the nested quality of physiological variability within individuals.

\section{Maternal Sensitivity as a Moderator of SNS and HPA Coordination}

It was hypothesized that maternal sensitivity moderates the relation between cortisol and salivary alpha-amylase, such that higher levels of sensitivity correspond to greater coordination between these biomarkers (and thus respective systems). Cortisol alone did not correlate with sAA (i.e., no main effect of cortisol), indicating that system coordination did not occur in a linear fashion for all infants at all times. The lack of 'average level coordination' (as termed by Laurent et al., 2013) is not surprising considering the dissociative functions of these systems and their 
overlapping excitatory and suppressive effects on one another across different stages of stress responding (Laurent et al., 2012; Sapolsky et al., 2000). Importantly, however, the significant two-way interaction between maternal sensitivity and cortisol revealed that coordination between these systems occurred in a non-linear fashion, such that these systems are coordinated (or uncoordinated) in the context of maternal sensitivity but not separately from this factor. Further, supporting the notion that system coordination is different at different times, the significant three-way interaction between maternal sensitivity, cortisol and sampling time indicated that the influence of maternal sensitivity on sAA/cortisol coordination varied according to the stage of responsivity (at baseline, peak or recovery). Across sampling time, infants of more sensitive mothers demonstrated greater coordination between their sAA and cortisol levels. At baseline and peak levels, higher levels of sensitivity were related to a positive relationship between sAA and cortisol, such that infants with higher levels of sAA also produced higher levels of cortisol. This finding was particularly robust for relations of sAA and cortisol at baseline (i.e., coordinated basal activity). Notably, for infants of less sensitive mothers, there was no apparent relationship between sAA and cortisol across baseline and peak time periods; higher levels of cortisol were not indicative of higher levels of sAA for these infants. During the recovery period, the sAA/cortisol slope for all infants became slightly negative, such that higher levels of cortisol were related to lower levels of sAA. This relationship was steeper for infants with more sensitive mothers, and suggests that sAA recovers at a faster speed that cortisol (i.e., cortisol levels may continue to be elevated while sAA has returned to baseline concentrations). The different pattern of coordination in the recovery period may relate to the physiological properties and effects of each system. For example, cortisol is known to produce counter-acting effects on the sympathetic response that facilitates SNS recovery (Boyce \& Ellis, 2005, Del Guidice et al., 
2011, Munck, Guyre, \& Holbrook, 1984). In addition, the HPA-axis is known to recover over a longer period of time than the SNS (Gunnar \& Quevedo, 2007). In the present study, 40 minutes may not have been sufficient to capture the prolonged HPA axis recovery period. Even so, the feasibility of collecting a 60-minute sample from infants is low considering the one hour feeding and drinking restrictions pre-stressor and post-stressor.

Coordination between systems is not expected to be fixed across all stressors and time. As such, coordination is not merely the synchrony between biomarker output but rather how the trajectories unfold across particular stressor demands. Similar to the definition of flexibility, different patterns of SNS and HPA coordination may be adaptive in different contexts, especially considering many stressors of lower threat do not activate the HPA-axis (Jansen et al., 2010). The present results did not show a significant four-way interaction between maternal sensitivity, time, cortisol and stressor, likely because sAA trajectories between low and high sensitivity infants were similar across stressors: infants of more sensitive mothers produced expected increases in sAA across peak time and decreases during recovery, whereas infants of less sensitive mothers had decreased levels of sAA during peak with increases during recovery. Even so, visual analysis of coordination patterns between stressors (Figure 1) revealed an important distinction of contextually-specific coordination between infants of high and low sensitivity. Infants of more sensitive mothers produced expected patterns of coordination in each stressor: when subjected to frustration in the TFP, these infants mounted an SNS response but not an HPA response; in the SSP, these infants mounted both SNS and HPA responses, with recovery occurring in both systems but with sAA demonstrated a steeper decline. This may further relate to the different direction of coordination seen in the recovery period of the three-way interaction. In the TFP with sensitive infants, higher levels of sAA during with recovery would be expected 
with lower levels of cortisol since the HPA is not activated during this stressor, whereas cortisol output is expected to be higher in the SSP where it is activated.

Our coordination results are consistent with the sensitive and buffered basal activity and responsivity profile patterns put forward in the adaptation calibration model (ACM; Del Guidice et al., 2011). Within the sensitive responsivity patterns of the ACM, children raised in sensitive, supportive environments are expected to have moderate baseline SNS and HPA activity and high SNS and HPA responsivity to stress (i.e., coordination in both systems at baseline and reactivity). This pattern was apparent in the present study for infants of more sensitive mothers: sAA and cortisol baseline levels were moderate in comparison to higher levels during peak reactivity. The ACM posits that this pattern of basal activity and responsivity is adaptive for children in sensitive environments as it allows them to react to occasional, brief stressors in their environment and makes them responsive to social and learning cues without any negative fitness costs (Del Guidice et al.). Children in less sensitive environments, however, may experience the buffered responsivity pattern, characterized by low-to-moderate SNS basal activity and reactivity and moderate HPA basal activity and responsivity. In the present study, infants of less sensitive mothers had significantly lower sAA reactivity to acute stress than their more sensitive counterparts. Reduced HPA reactivity in response to stress for less sensitive infants was also found previously (Atkinson et al., 2013). Reductions in basal activity and reactivity in these systems may be optimal in more stressful environments where stressors may happen more regularly and for longer periods, therefore increasing the cost of producing high levels of catecholamines or glucocorticoids (Del Guidice et al.). The frequency of stressors in these environments may be elevated because insensitive parenting interactions can themselves be stressors for infants (Belsky \& Pleuss, 2009). The presence of increased sAA during the recovery 
period for infants of less sensitive mothers in this study may be evidence of this as the recovery period represents a period of unstructured play time for the dyad. As such, unstructured play appears to have been more stressful for these infants compared to the structured challenge.

Del Guidice and colleagues' (2011) environment-specific explanation of coordination has roots in the differential-susceptibility hypothesis, which suggests that one's genetic makeup can interact with environmental conditions to predict child outcomes (Belsky \& Pleuss, 2009). This hypothesis was previously supported in the present infant sample in regards to dopamine-related genes, maternal depression and cortisol reactivity (Ludmer et al., 2015). The genetic underpinnings of SNS reactivity and environmental contexts have not been explored in motherinfant dyads, though research from adult samples suggests that genetic variants may moderate cardiac reactivity in response to psychological stress (e.g., the serotonin transporter gene 5HTTLPR long allele; Williams et al., 2008). Exploration of genetic moderators and mediators may help contextualize the current coordination findings.

The pattern of coordination partially fits with Andrew and colleagues' (2013) stress coherence/compensation model. The present study showed that infants of more sensitive mothers demonstrated similar levels of sAA and cortisol where expected (in response to separation). However, infants of less sensitive mothers did not demonstrate compensation between their systems as predicted by Andrews and colleagues. For example, in the TFP, less sensitive infants failed to mount an SNS response to the stressor yet their HPA-axis remained unresponsive. According to the stress coherence/compensation model, the HPA axis should have compensated for the lack of SNS responding, which it did not. Of note, this model includes the importance of measuring additional stress systems such as the parasympathetic nervous system, which was not assessed here. Further, the authors emphasize perceived threat of stress (i.e., cognitive appraisal) 
as an important 'third stress system', though our ability to measure this facet in infants is limited. Nevertheless, further examination of the model with additional stressors and developmental factors is warranted.

The coordination results are also similar to the interactive model put forward by Bauer and colleagues (2002), which predicts that synchrony between stress systems (e.g., high sAA and high cortisol in response to potent stressors) is indicative of adaptive physiology in development. However, an essential tenet of Bauer et al.'s model is that the synchrony of systems can be used to predict whether a child is at low or high risk for displaying internalizing and externalizing disorders. Though this information was not feasible to collect in a sample of infants, it is fortunate that the present sample is part of an ongoing longitudinal study where there is the possibility to compare follow-up measurements of behaviour to patterns of physiology seen in early childhood. Based on their model, it would be expected that infants who displayed greater system synchrony (i.e., those with sensitive mothers) would have fewer problematic internalizing or externalizing features. Findings of this nature would help explain the mechanism of early developmental experiences and later psychopathology.

As mentioned, coordination between sAA and cortisol in infants of more sensitive mothers was most robust at baseline (i.e., pre-stressor). One potential mechanism for greater levels of basal SNS and HPA activity in sensitive infants is the presence of physiological attunement between mothers and infants. Attunement refers to the synchrony of stress responses across dyadic partners (Field, 1994; Feldman, 2007). Maternal cortisol concentrations for this sample of infants had been previously assayed and were found to correlate with infant baseline levels, i.e., sensitive mothers and their infants had higher baseline cortisol concentrations (Atkinson et al., 2013). The correlation between sensitive mother-infant baseline and slope 
concentrations was given as evidence of HPA-axis attunement within this sample of dyads (Atkinson et al.). To extend the current findings, maternal concentrations of sAA must be assayed and analyzed to determine the presence of attunement in mother-infant baseline sAA. Attunement of mother-infant baseline sAA has been found in other studies (Kivlighan, 2006; Laurent et al., 2012), though there is mixed evidence for whether sAA attunement is maintained during the reactivity and recovery stages; Laurent and colleagues found synchronous patterns of mother-infant sAA in response to an infant emotion-eliciting stressor, whereas Kivlighan did not find significant synchrony in response to a similar paradigm. Neither study examined the influence of maternal sensitivity on mother-infant SNS attunement, indicating an avenue of future research.

In sum, the present study found evidence of maternal sensitivity as a moderator between infant sAA and cortisol. As predicted, maternal sensitivity was found to moderate sAA/cortisol coordination at baseline and reactivity time periods, such that higher but not lower levels of sensitivity related to increased synchrony in biomarker output.

\section{Maternal Sensitivity as a Moderator of Infant SNS Flexibility}

The second primary hypothesis predicted that maternal sensitivity moderates infant sAA flexibility across stressors, such that higher levels of maternal sensitivity are related to greater flexibility within infants. Results demonstrated that sAA output varied according to the type of stressor: the strange situation procedure produced higher sAA trajectories on average compared to the toy frustration procedure. Therefore, overall variability of the SNS response across infants was demonstrated (though in an unexpected fashion that will be discussed below). However, as discussed previously, variability is not necessarily equated with flexibility as flexibility is context-dependent (Atkinson et al., in press). Previously in this sample, maternal sensitivity was 
found to moderate cortisol flexibility (Atkinson et al., 2013), with infants of more sensitive mothers mounting a more robust response to the strange situation procedure compared to the toy frustration procedure. Analyses in the present study, however, found no significant interactions between stressor and maternal sensitivity; though from a sample size perspective, the current study had one third of the power than the previous investigation. Explanations for this null finding may relate to methodology and the differences in SNS physiology compared to the HPA axis.

From a methodological perspective, the null finding may be a product of the selected stressor tasks. In developmental contexts, far more information is available on what components are more effective in eliciting an adrenocortical response (Dickerson \& Kemeny, 2004; Gunnar et al., 2009; Jansen et al., 2010). Meta-analytic or systematic reviews on the effectiveness of stressor paradigms on SNS reactivity, however, are not available. Even in the current study, the stressors were originally designed to capitalize on their differential potency for eliciting cortisol reactivity, with sympathetic effects a secondary consideration. Thus, future studies may benefit from the selection of different paradigms. For example, in adult samples, physical exercise paradigms (e.g., ergometer tests) are particularly potent for eliciting SNS responses, above and beyond psychological stressors (Skoluda et al., 2015). Ergometer tests may be impractical in infant samples but another substitute physical-activity-based task may be more effective in testing the SNS flexibility hypothesis and how it relates to maternal sensitivity.

Despite the lack of support for a maternal sensitivity and challenge interaction, the pattern of SNS variability uncovered in the present study is worth unpacking. Contrary to prior literature emphasizing the effectiveness of effort and/or frustration on eliciting SNS responsivity (Frankenhaesuer, 1982; Laurent et al., 2012; Lundberg \& Frankenhaeuser, 1980; Ursin, Baade, 
\& Levine, 1978), we found that sAA reactivity was greater in response to relational threat than toy frustration. This finding is incongruous with other infant stress studies. For example, Laurent and colleagues (2011) found elevated sAA in response to challenge tasks compared to a separation stressor. Mechanistically, the discrepancy between our finding and others may be the product of: 1) differences in stressor components; or 2) differences in sampling time. In regard to stressor differences, we used a specific, single toy withdrawal procedure to induce infant frustration. Other studies have used a combination of effortful, frustrating and novel tasks, most commonly from the Laboratory Temperament Assessment Battery (LAB-TAB; Goldsmith \& Rothbart, 1996). For example, Kivlighan (2006) collected 6-month old infant saliva samples before, and 20 and 40 minutes following the combination of the LAB-TAB tasks. Additionally, Laurent and colleagues (2012) used the Fear, Frustration and Joy LAB-TAB tasks to elicit emotion-specific forms of stress. They also administered the LAB-TAB directly after a clean-up task without a separating rest/recovery period and collected saliva immediately post stressor and 15-minutes following. Thus, comparing sAA findings between these studies is made difficult due to methodological inconsistencies. Future studies examining sAA flexibility and variability may benefit from the separation of the different components elicited in the LAB-TAB (e.g., separate examination of novelty, emotionality).

In regard to the second methodological difficulty, the aforementioned studies all used different salivary sampling times to capture sAA reactivity. Salivary alpha-amylase output peaks within the first 5 to 10 minutes of inducing acute stress and it is recommended to continue measuring it at 10 minute intervals until 20 to 30-minutes post-stressor (Rohleder \& Nater, 2009; Takai et al., 2004). Yet, studies are often designed with the primary intention of measuring cortisol trajectories and, as such, salivary sampling times are preferentially selected to capture 
cortisol's peak that occurs approximately 20-minutes post-stressor (Dickerson \& Kemeny, 2004). Sample timing in the present study was specifically designed to capture both sAA and cortisol. Furthermore, we excluded samples that were collected outside of the expected peak and recovery windows in order to confidently assess trajectories. Other studies, such as Kivlighan's (2006) dissertation, may have missed sAA's peak response given that the first sampling time occurred 20-minutes post-stressor. An additional possibility is that infants display variability in the timing of their SNS peak reactivity. For example, in a sample of 27 infants aged 12 to 18 months, Goldberg and colleagues (2003) found that half of these infants produced peak cortisol at 20 minutes and the other half peaked at 40 minutes. It is unknown whether a temporal group-level distinction occurs in infant sAA responding and what infant or environmental characteristics would influence this variability. Future studies that apply greater consistency in sample timing or included additional sample times are required to clarify these inconsistencies.

\section{Interactions between coordination and flexibility}

The lack of support for the flexibility hypothesis negated the third hypothesis, which sought to explore potential interactions between coordination and flexibility. No significant interactions between stressor and maternal sensitivity, cortisol reactivity, or sampling time were found. However, as discussed in relation to coordination, different patterns of sAA/cortisol coordination were witnessed visually in the profiles of infants of high versus low sensitivity. Power to detect this potential interaction will increase with attention to the substantive and methodological considerations discussed above. Therefore, this explorative hypothesis should remain in future studies that examine parenting behaviours and infant stress physiology. 


\section{Sex Differences in Salivary Alpha-Amylase Trajectories}

Sympathetic sex differences were not predicted a priori. However, the inclusion of sex as a covariate revealed significant influences on overall levels of infant sAA output (main effect of sex) and sAA trajectories (interaction between time and sex). On average, female infants had higher overall levels of sAA and greater sAA at peak and recovery time periods. In comparison, male infants had lower, relatively stable levels of sAA output on average. Developmental research on sympathetic sex differences is limited but most of the studies available have not found significant SNS sex differences. Davis and Granger (2009) found no main effect or interaction effects of sex with time in a sample of infants measured at 2, 6, 12 and 24 months. Further, Hill-Soderlund et al. (2008) found no sex differences in sAA responding in infants 13 to 14-months-of-age following the strange situation procedure. No sex differences in toddler (12 to 24 months) sAA awakening response have been found either (Bright, Frick, Out, \& Douglas, 2014). In a sample of trauma-exposed adolescents (12 to 19-year-olds who experienced Hurricane Katrina), sex and sAA were only found to interact in relation to depression, such that females with higher sAA levels had higher levels of depressive symptoms in comparison to males (Vigil et al., 2010). The adaptive calibration model posits that sex differences in sympathetic and adrenocortical responding will be most prominent in infants exposed to chronically stressful or traumatic environments, such that males in these circumstances will demonstrate patterns of blunted responsivity (Del Giudice et al., 2011). However, preliminary evidence from the theory's developers found only partial support for these predictions, with female children overrepresented in the vigilant stress classification (i.e., high SNS and HPA responsivity) but no elevation of males in the buffered or unemotional categories (Del Giudice, Hinnant, Ellis, \& El Sheikh, 2012). In the present study, sex did not interact with maternal 
sensitivity to predict sAA (or cortisol) responsivity as the adaptive calibration model would suggest (though importantly the vigilant pattern could not be assessed in our study as we did not assess parasympathetic activity). Thus, while the present findings regarding sympathetic sex differences are interesting, they require replication in future studies and should be explored in the context of known moderators of gender, such as temperament or emotion regulation.

Other theories of sex differences in physiological, emotional and behavioural stress responding exist (e.g., Taylor et al., 2000; Vigil, 2009). For example, the 'tend-and-befriend' model proposed by Taylor and colleagues (2000) suggests that females will engage in tending (e.g., soothing and quieting offspring) and befriending (e.g., activation of social networks) when exposed to threat, as opposed to the traditional 'fight-or-flight' responses. Oxytocin, a hormone related to attachment behaviours, has been proposed as a mechanism for these differential stress responses in females. It is unclear whether these differences exist in infants and children as research linking sex, behaviour and physiology in these populations is lacking. In support of the tend-and-befriend theory, David and Lyon-Ruth (2005) found that female infants with disorganized attachments engaged in more approach behaviours than male infants following frightening maternal behaviours, however, they did not include measures of physiological responses. In a previous investigation with the present sample, no sex differences were found between infant cortisol responses and emotion-regulation strategies (Khoury et al., 2015), but this was not explored in regard to sAA. Without knowing whether female infants engaging in tend-and-befriend behaviours have distinct patterns of sympathetic responding, we cannot contextualize the present study's sAA sex differences using this theory. Thus, future exploration of the interaction between sAA (and other SNS measures), oxytocin and infant care-seeking behaviours during acute stress are needed. 


\section{Applied Implications}

In addition to the theoretical implications of this research, our findings have relevance for child health interventions and stress research methodology. We found that infants of more sensitive mothers had more adaptive patterns of stress system coordination and produced sAA/cortisol responses that better matched the demands of the stressor, compared to infants of less sensitive mothers. Thus, quality of early care was found to moderate important components of stress physiology that may put infants of less sensitive mothers at greater risk of negative health and behavioural outcomes; or to quote the familiar phrase, provide a mechanism for how less sensitive environments "get under the skin" to produce unfavourable child outcomes. The adverse consequences of dysregulated HPA-axis responding are well-established in the literature (Chrousos, 2009; Danese \& McEwen, 2012; Gunnar \& Quevedo, 2007; McEwen \& Gianaros, 2011; Miller, Chen, \& Zhou, 2007) but the present findings highlight the need to explore how dysregulation in additional stress systems and their interconnectivity are associated with developmental outcomes. From a public health perspective, these results highlight the need for parenting interventions that increase sensitivity and strengthen the caregiver-child bond.

Fortunately, parenting interventions specifically designed to enhance maternal sensitivity are available. A meta-analysis by Bakermans-Kranenburg and colleagues (2003) found that parental sensitivity interventions across 88 studies were effective at increasing parental sensitivity on average $(d=.33)$. No experimental studies have specifically tested whether these interventions would translate to enhanced or reparative changes in infant stress physiology, though this would be a meaningful avenue of future research. 


\section{Study Limitations and Future Directions}

Additional limitations of the present study warrant discussion. First, the sample is comprised of low-risk community dyads who were mostly Caucasian, middle-to-upper class, and co-parenting. Though our sample had adequate variability in maternal sensitivity, it would be interesting to explore the forwarded hypotheses in an at-risk sample. For example, Kivlighan (2006) found evidence of SNS/HPA reactivity and recovery coordination is a sample of lowincome dyads. Replication in samples of greater diversity (e.g., ethnic background, socioeconomic status) or clinical populations (e.g., mothers with depression) should be conducted to widen the generalizability of these findings. Second, the influence of infant temperament was not included in the present study. Previous studies assessing mother-dyad SNS/HPA-axis attunement and individual patterns of reactivity have demonstrated relations between patterns of synchrony and temperament styles (Laurent et al., 2012; Kivlighan, 2006). Thus, this important infant characteristic should be considered in future studies. Third, though this study includes measures from both the HPA-axis and the SNS, there are additional systems that comprise the comprehensive stress response. These include aspects of the immune system, as well as the parasympathetic nervous system that exerts suppressive effects on the SNS to obtain homeostasis. In order to build our understanding of comprehensive stress development, studies must build measures of these systems into their methodologies.

\section{Conclusion}

In conclusion, we found evidence that maternal sensitivity moderates the coordination between infant sAA and cortisol basal activity and reactivity in response to acute stress. Across baseline and peak sampling periods, higher levels of maternal sensitivity were associated with positive relations between sAA and cortisol, such that higher levels of cortisol were associated 
with higher levels of sAA. At lower levels of sensitivity, infant cortisol and sAA levels were unrelated (i.e., no evidence of coordination). Infants demonstrated variable SNS responses to the different stressor challenges, with higher reactivity in the strange situation than the toy frustration procedure. However, maternal sensitivity did not significantly moderate the level of flexibility within this system, as had been found previously with cortisol in this sample (Atkinson et al., 2013), though visual trends toward increased sAA flexibility were witnessed. Overall, these results provide support for the prediction that maternal sensitivity relates to important features of stress physiology, namely SNS/HPA baseline and reactivity coordination and SNS variability, in low-risk community samples. 
Table 1

Raw means, standard deviations, medians and interquartile ranges of infant salivary alphaamylase (sAA) and cortisol concentrations and average maternal sensitivity from the Maternal Behaviour Q-Sort

\begin{tabular}{|c|c|c|c|c|c|c|c|c|}
\hline \multirow[b]{2}{*}{ Variable } & \multicolumn{4}{|c|}{ Toy Frustration Procedure } & \multicolumn{4}{|c|}{ Strange Situation Procedure } \\
\hline & $M$ & $S D$ & Median & $I Q R$ & $M$ & $S D$ & Median & $I Q R$ \\
\hline $\begin{array}{l}\text { 1. sAA } \\
\text { baseline }\end{array}$ & 66.20 & 42.14 & 55.43 & 49.90 & 91.72 & 68.42 & 71.26 & 93.21 \\
\hline $\begin{array}{l}\text { 2. sAA } 5 \\
\text { minutes }\end{array}$ & 69.79 & 42.87 & 61.58 & 56.25 & 90.19 & 65.55 & 73.96 & 87.88 \\
\hline $\begin{array}{l}\text { 3. sAA } 20 \\
\text { minutes }\end{array}$ & 74.31 & 48.56 & 61.66 & 60.23 & 85.55 & 58.43 & 66.50 & 77.86 \\
\hline $\begin{array}{l}\text { 4. Cortisol } \\
\text { baseline }\end{array}$ & 4.25 & 3.02 & 3.83 & 2.57 & 4.70 & 4.42 & 3.52 & 2.66 \\
\hline $\begin{array}{l}\text { 5. Cortisol } 20 \\
\text { minutes }\end{array}$ & 3.66 & 3.01 & 2.91 & 1.96 & 5.10 & 4.42 & 3.63 & 3.23 \\
\hline $\begin{array}{l}\text { 6. Cortisol } 40 \\
\text { minutes }\end{array}$ & 3.46 & 2.17 & 2.93 & 2.30 & 4.85 & 3.85 & 3.81 & 2.79 \\
\hline $\begin{array}{l}\text { 7. Maternal } \\
\text { sensitivity }\end{array}$ & .47 & .29 & & & & & & \\
\hline
\end{tabular}


Table 2

Fixed effect estimates from the 3-level multilevel model predicting log transformed salivary alpha-amylase levels

\begin{tabular}{lccc}
\hline Fixed Effect & $B$ & $S E$ & $t$ \\
\hline Intercept $_{\text {Sex of Infant }}^{\mathrm{a}}$ & 1.68 & .036 & $47.35^{* * *}$ \\
Maternal sensitivity $^{\mathrm{b}}$ & .11 & .049 & $2.24^{*}$ \\
Time $^{\mathrm{b}}$ & -.0063 & .085 & -.075 \\
Challenge $^{\mathrm{c}}$ & .0046 & .0013 & .34 \\
Cortisol $^{\mathrm{d}}$ & .097 & .018 & $5.38^{* * *}$ \\
Sex x Time $_{\text {Time x maternal sensitivity }}$ & -.013 & .034 & -.39 \\
Cortisol x maternal sensitivity & .021 & .010 & $2.07^{*}$ \\
Time x cortisol & .076 & .041 & $1.84^{\ddagger}$ \\
Time x cortisol x maternal sensitivity & .29 & .11 & $2.55^{* *}$ \\
\hline
\end{tabular}

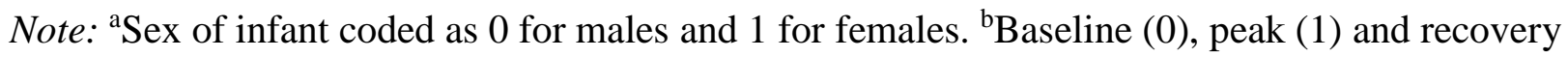
(2) sampling periods (sAA = baseline, 5 minutes, 20 minutes; cortisol = baseline, 20 minutes, 40 minutes). ${ }^{c}$ Stressor dummy-coded as $0=$ toy frustration procedure and $1=$ strange situation. ${ }^{d} \log$ transformed cortisol values. $* p<.05 * p<.01 * * p<.001 * * * \neq p=.067$ 


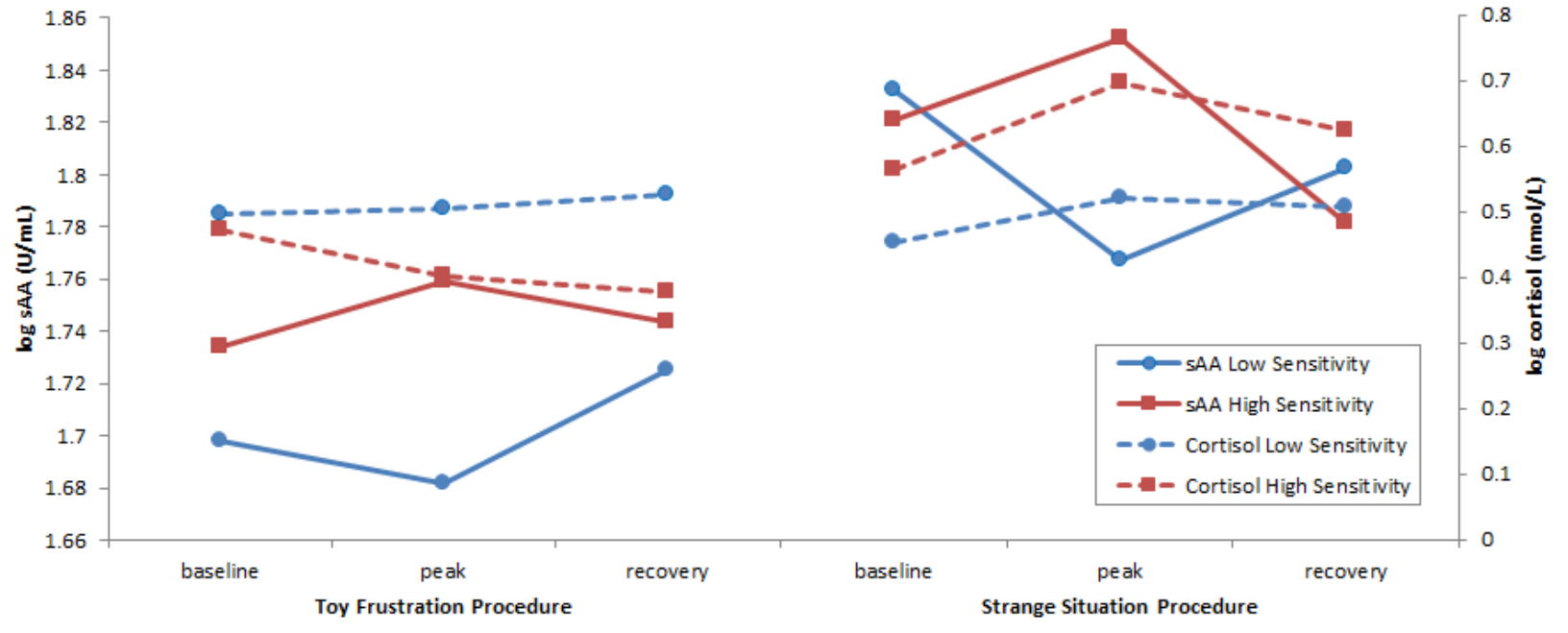

Figure 1. Patterns of log transformed sAA and cortisol across stressors (TFP and SSP), and time, at high and low levels of maternal sensitivity ( \pm 1SD). 


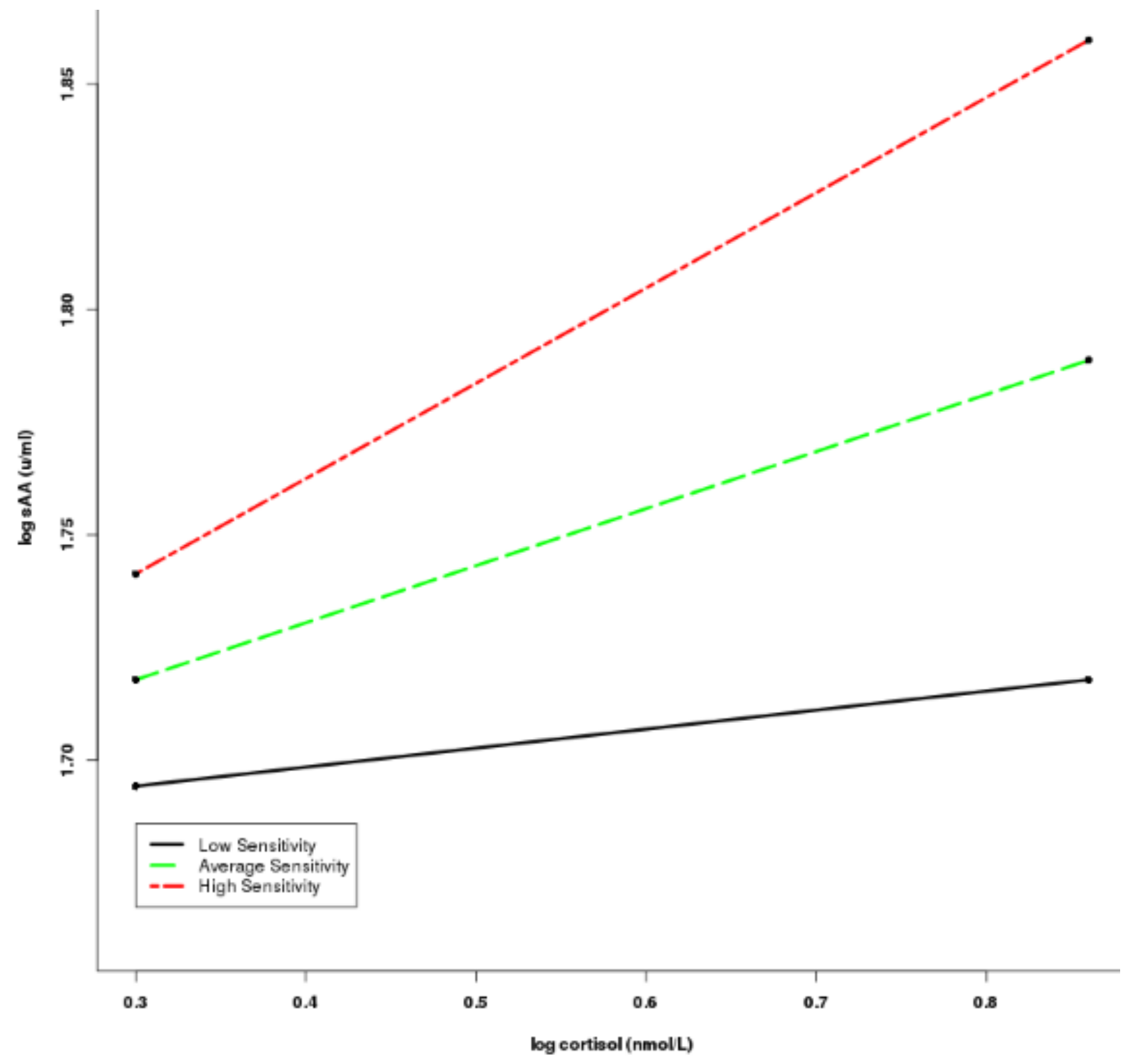

Figure 2. 2-way interaction between maternal sensitivity and log transformed total cortisol output (i.e., cortisol collapsed across time and stressor) to predict overall levels of log transformed salivary alpha-amylase. Levels of maternal sensitivity represent -1 SD, mean, and $+1 \mathrm{SD}$. Higher levels of maternal sensitivity are associated with greater coordination between cortisol and salivary alpha-amylase. 


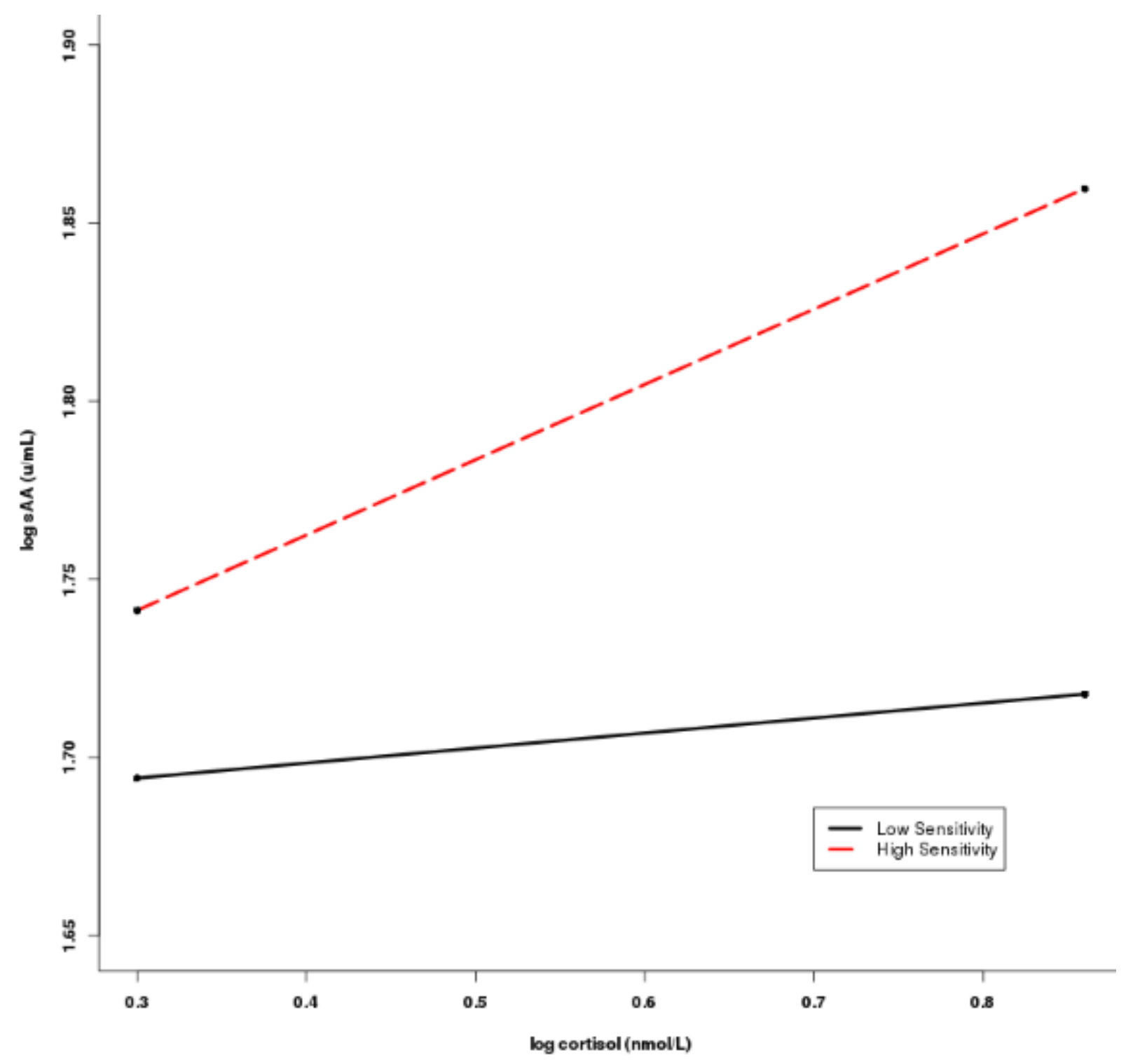

Figure 3. Baseline (5-minutes pre-stressor) coordination of log transformed salivary alphaamylase and cortisol at high and low levels of maternal sensitivity ( $\pm 1 \mathrm{SD})$. Infants of more sensitive mothers had significantly greater baseline sAA and cortisol coordination (i.e., sAA increases as cortisol increases) compared to infants of less sensitive mothers. 


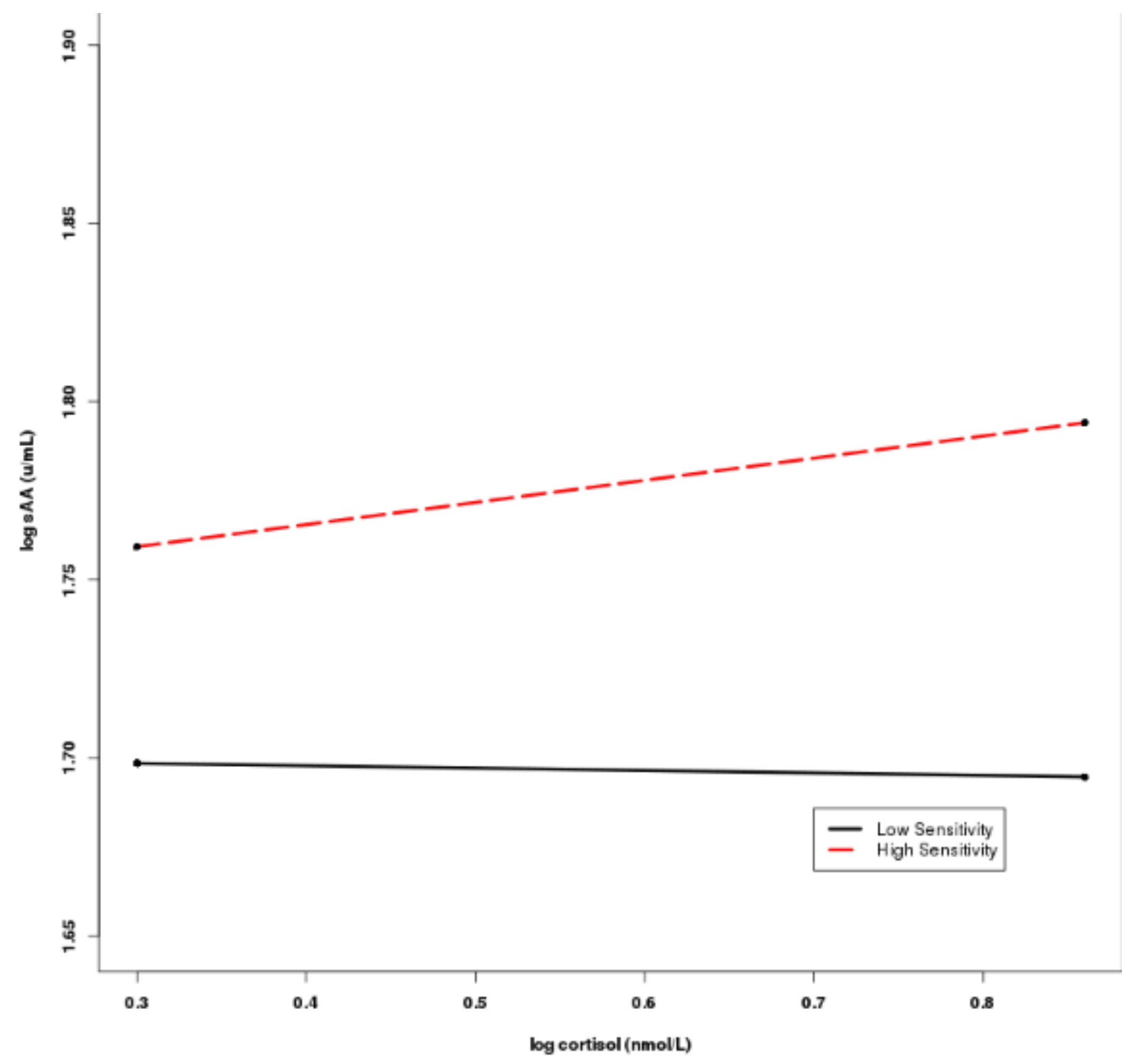

Figure 4. Peak sampling time coordination of log transformed salivary alpha-amylase and cortisol at high and low levels of maternal sensitivity ( $\pm 1 \mathrm{SD}$ ). Infants of more sensitive mothers show greater coordination of sAA and cortisol during peak reactivity compared to infants of mothers with lower sensitivity. Peak time represents the +5 minute sample for sAA and the +20 sample for cortisol. 


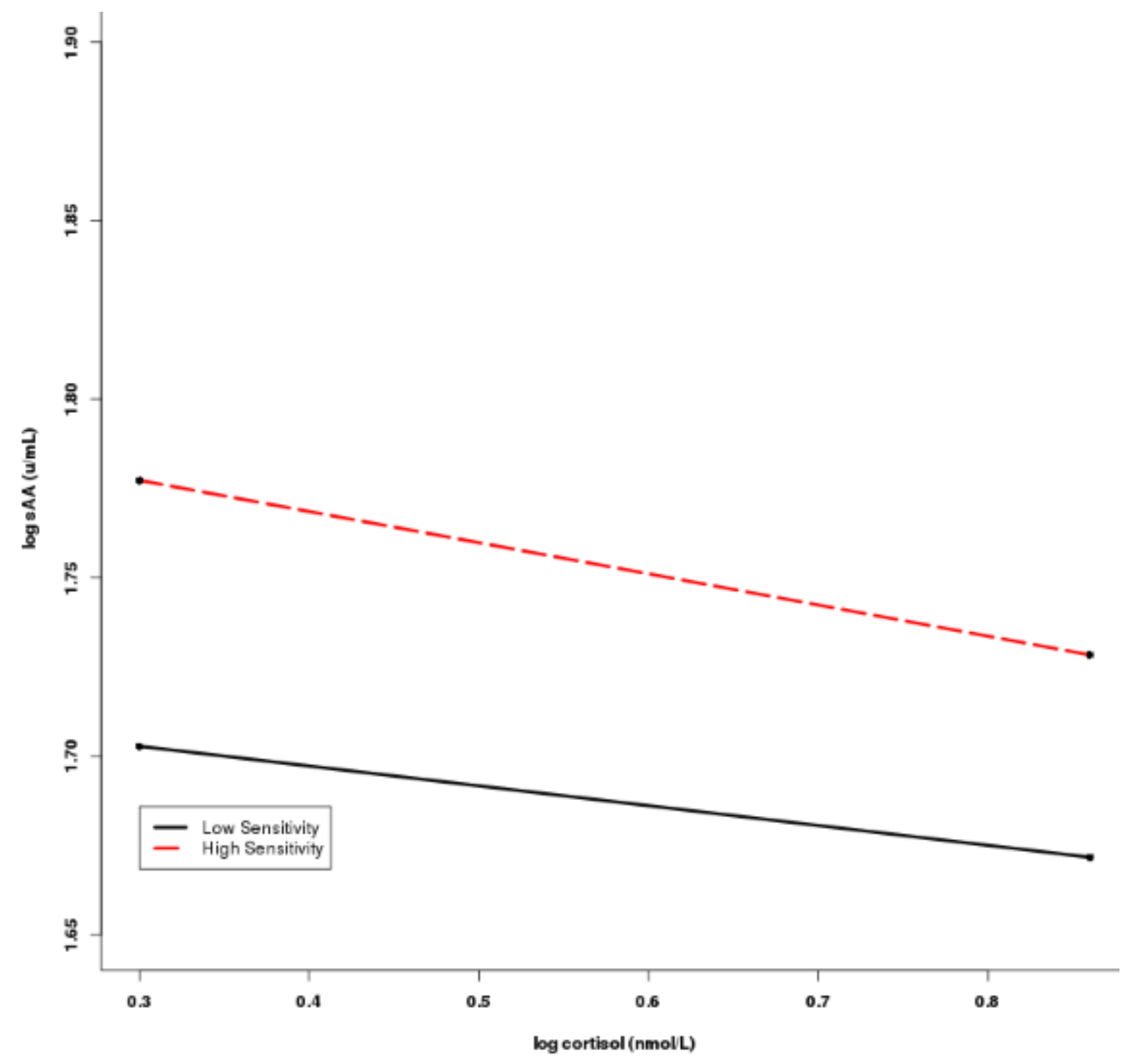

Figure 5. Recovery sampling time coordination of log transformed salivary alpha-amylase and cortisol at high and low levels of maternal sensitivity ( $\pm 1 \mathrm{SD}$ ). Higher levels of salivary alphaamylase are associated with lower levels of cortisol and vice versa, particularly for infants of more sensitive mothers. Recovery represents the +20 minute sample for sAA and the +40 sample for cortisol. 


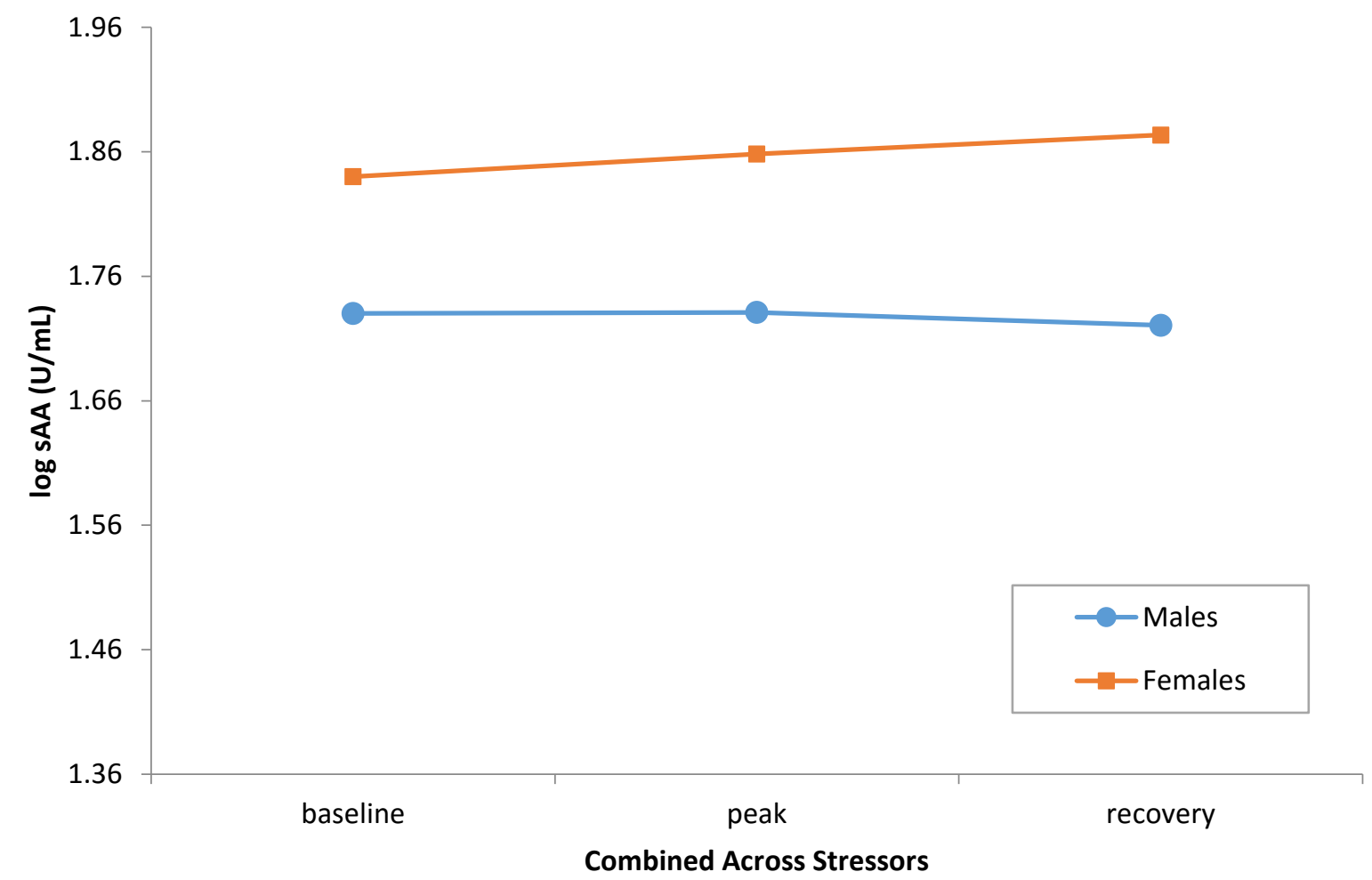

Figure 6. 2-way interaction between sampling time and sex to predict log salivary alphaamylase, such that female infants had higher levels of sAA on average compared to males, particularly at peak and recovery time points. Note that sampling time is collapsed across stressors. 


\section{References}

Aiken, L. S., \& West, S. G. (1991). Multiple regression: Testing and interpreting interactions. Newbury Park, CA: Sage Publications.

Ainsworth, M. D. S., Blehar, M. C., Waters, E., \& Wall, S. (1978). Patterns of attachment: A psychological study of the strange situation. Hillside, NJ: Lawrence Erlbaum Associates.

Ali, N., \& Pruessner, J. C. (2012). The salivary alpha amylase over cortisol ratio as a marker to assess dysregulations of the stress systems. Physiology \& Behaviour, 106, 65-72. doi:10.1016/j.physbeh.2011.10.003

Andrews, J., Ali, N., \& Pruessner, J. C. (2013). Reflections on the interaction of psychogenic stress systems in humans: The stress coherence/compensation model. Psychoneuroendocrinology, 38(7), 947-961. doi:10.1016/j.psyneuen.2013.02.010

Atkinson, L., Khoury, J., Ludmer, J., Jamieson, B., \& Gonzalez, A. (In press). Stress physiology in infancy and early childhood: Cortisol flexibility, attunement, and coordination. Journal of Neuroendocrinology.

Atkinson, L., Gonzalez, A., Kashy, D. A., Santo Basile, V., Masellis, M., Pereira, J., ... Levitan, R. (2013). Maternal sensitivity and infant and mother adrenocortical function across challenges. Psychoneuroendocrinology, 38(12), 2943-2951.

doi:10.1016/j.psyneuen.2013.08.001

Atkinson, L., Paglia, A., Coolbear, J., Niccols, A., Poulton, L., Leung, E., \& Chisholm, V. C. (2000). Assessing maternal sensitivity in the context of attachment security: A metaanalysis. In G. M. Tarabulsy, S. Larose, D. R. Pederson \& G. Moran (Eds.), Attachment and Development: The Role of First Relationships in Human Development (pp. 27-56). Presses de l'Universite du Quebec, Quebec.

Bakermans-Kranenburg, M. J., Van IJzendoorn, M. H., \& Juffer, F. (2003). Less is more: Metaanalyses of sensitivity and attachment interventions in early childhood. Psychological bulletin, 129(2), 195.

Bauer, A. M., Quas, J. A., \& Boyce, W. T. (2002). Associations between physiological reactivity and children's behaviour: Advantages of a multisystem approach. Developmental and Behavioural Prediatrics, 23(2), 102-113.

Belsky, J., \& Pluess, M. (2009). Beyond diathesis stress: differential susceptibility to environmental influences. Psychological bulletin, 135(6), 885. doi: 10.1037/a0017376 
Black, P. H., \& Garbut, L. D. (2002). Stress, inflammation and cardiovascular disease. Journal of Psychosomatic Research, 52(1), 1-23.

Blair, C., Granger, D. A., Kivlighan, K. T., Mills-Koonce, R., Willoughby, M., Greenberg, M. T., ... \& Fortunato, C. K. (2008). Maternal and child contributions to cortisol response to emotional arousal in young children from low-income, rural communities. Developmental Psychology, 44(4), 1095-1109.

Blair, C., Granger, D. A., Willoughby, M., \& Kivlighan, K. T. (2006). Maternal sensitivity is related to hypothalamic-pituitary-adrenal axis stress reactivity and regulation in response to emotion challenge in 6-month-old infants. Annals of the New York Academy of Sciences, 1094 (1), 263-267.

Bosch, J. A., Veerman, E. C. I., de Geus, E. J., \& Proctor, G. B. (2011). $\alpha$-Amylase as a reliable and convenient measure of sympathetic activity: don't start salivating just yet! Psychoneuroendocrinology, 36(4), 449-453. doi: 10.1016/j.psyneuen.2010.12.019

Boyce, W. T., \& Ellis, B. J. (2005). Biological sensitivity to context: I. An evolutionarydevelopmental theory of the origins and functions of stress reactivity. Developmental Psychopathology, 17, 271-301.

Braungart-Rieker, J. M., \& Stifter, C. A. (1996). Infants' responses to frustrating situations: Continuity and change in reactivity and regulation. Child Development, 67, 1767-1779.

Bright, M. A., Frick, J. E., Out, D., \& Granger, D. A. (2014). Individual differences in the cortisol and salivary $\alpha$-amylase awakening responses in early childhood: Relations to age, sex, and sleep. Developmental psychobiology, 56(6), 1300-1315.

Chatterton Jr., R. T., Vogelsong, K. M., Lu, Y.C., \& Hudgens, G. A. (1996). Salivary alphaamylase as a measure of endogenous adrenergic activity. Clinical Physiology, 16, 433-448.

Chen, F. R., Raine, A., \& Granger, D. A. (2015). Tactics for modeling multiple salivary analyte data in relation to behaviour problems: Additive, ratio and interaction effects. Psychoneuroendocrinology, 51, 188-200. doi: 10.1016/j.psyneuen.2014.09.027

Chrousos, G. P. (2009). Stress and disorders of the stress system. Nature Reviews Endocrinology, 5(7), 374-381.

Cohen, J., Cohen, P., West, S. G., Aiken, L. S. (2003). Applied multiple regression/correlation analysis for the behavioural sciences ( $3^{\text {rd }}$ Ed.). Mahwah, NJ: Lawrence Erlbaum Associates. 
Danese, A., \& McEwen, B. S. (2012). Adverse childhood experiences, allostasis, allostatic load, and age-related disease. Physiology \& Behaviour, 106, 29-

39. doi:10.1016/j.physbeh.2011.08.019

David, D. H., \& Lyons-Ruth, K. (2005). Differential attachment responses of male and female infants to frightening maternal behaviour: tend or friend versus fight or flight? Infant Mental Health Journal, 26(1), 1-18. doi: 10.1002/imhj.20033

Davis, E. P., \& Granger, D. A. (2009). Developmental differences in infant salivary alphaamylase and cortisol responses to stress. Psychoneuroendocrinology, 34, 795-804. doi: 10.1016/j.psyneuen.2009.02.001

de Kloet, E. R. (1991). Brain corticosteroid receptor balance and homeostatic control. Frontiers in Neuroendocrinology, 12(2), 95-164.

Del Guidice, M., Ellis, B. J., Shirtcliff, E. A. (2011). The Adaptive Calibration Model of stress responsivity. Neuroscience and Biobehavioural Reviews, 35, 1562-1592. doi: 10.1016/j.neubiorev.2010.11.007

Del Giudice, M., Hinnant, J. B., Ellis, B. J., \& El-Sheikh, M. (2012). Adaptive patterns of stress responsivity: a preliminary investigation. Developmental psychology, 48(3), 775-790. doi: $10.1037 / \mathrm{a} 0026519$

Dickerson, S. S., \& Kemeny, M. E. (2004). Acute stressors and cortisol responses: A theoretical integration and synthesis of laboratory research. Psychological Bulletin, 130, 355-391.

Dvorak, R. V., Callés-Escandon, J., \& Poehlman, E. T. (1998). Measurement and prediction of sympathetic nervous system activity in humans. Life Sciences, 62(16), 1397-1406.

El-Sheikh, M., Erath, S. A., Buckhalt, J. A., Granger, D. A., \& Mize, J. (2008). Cortisol and children's adjustment: The moderating role of sympathetic nervous system activity. Journal of Abnormal Child Psychology, 36, 601-611. doi: 10.1007/s10802-007-9204-6

Ellis, B. J., \& Del Giudice, M. (2014). Beyond allostatic load: Rethinking the role of stress in regulating human development. Development and Psychopathology, 26(01), 1-20. doi:10.1017/S0954579413000849

Engert, V., Vogel, S., Efanov, S. I., Duchesne, A., Corbo, V., Ali, N., \& Pruessner, J. C. (2011). Investigation into the cross-correlation of salivary cortisol and alpha-amylase responses to psychological stress. Psychoneuroendocrinology, 36, 1294-1302.

doi:10.1016/j.psyneuen.2011.02.018 
Epel, E. S., Blackburn, E. H., Lin, J., Dhabhar, F. S., Alder, N. E., Morrow, J. D., \& Cawthon, R. M. (2004). Accelerated telomere shortening in response to life stress. National Academy of Science, 101(49), 17312-17315.

Feldman, R., Singer, M., \& Zagoory, O. (2010). Touch attenuates infants’ physiological reactivity to stress. Developmental Science, 13, 271-278.

Fortunato, C. K., Dribin, A. E., Granger, D. A., \& Buss, K. A. (2008). Salivary alpha-amylase and cortisol in toddlers: Differential relations to affective behaviours. Developmental Psychobiology, 50, 807-818. doi: 10.1002/dev.20326

Frankenhaeuser, M. (1982). Challenge-control interaction as reflected in sympathetic-adrenal and pituitary-adrenal activity: comparison between the sexes. Scandinavian Journal of Psychology, Suppl. 1, 158-164.

Garson, D. G. (2013). Hierarchical linear modeling guide: Guide and applications. Thousand Oaks, CA: Sage Publications.

Gibson, A. (1981). The influence of endocrine hormones on the automatic nervous system. Journal of Autonomic Pharmacology, 1, 331-340.

Goldberg, S., Levitan, R., Leung, E., Masellis, M., Basile, V, Nemeroff, C. B., \& Atkinson, L. (2003). Stability of baseline and stress cortisol concentrations in 12-18-month-old infants: issues of time, location, and stressor. Biological Psychiatry, 54, 719-726.

Goldsmith, H. H., \& Rothbart, M. K. (1999). The Laboratory Temperament Assessment Battery. Madison, WI: University of Wisconsin-Madison.

Gordis, E. B., Granger, D. A., Susman, E. J., \& Tickett, P. K. (2006). Asymmetry between salivary cortisol and $\alpha$-amylase reactivity to stress: Relation to aggressive behaviour in adolescents. Psychoneuroendocrinology, 31, 976-987. doi:10.1016/j.psyneuen.2006.05.010

Gordis, E. B., Granger, D. A., Susman, E. J., \& Trickett, P. K. (2008). Salivary alpha amylasecortisol asymmetry in maltreated youth. Hormones and Behavior, 53(1), 96-103. doi:10.1016/j.yhbeh.2007.09.002

Granger, D. A., Kivlighan, K. T., Blair, C., El-Sheikh, M., Mize, J., Lisonbee, J. A., . . . Schwartz, E. B. (2006). Integrating the measurement of salivary $\alpha$-amylase into studies of child health, development, and social relationships. Journal of Social and Personal Relationships, 23(2), 267-290. doi: http://dx.doi.org/10.1177/0265407506062479 
Granger, D. A., Kivlighan, K. T., El-Sheikh, M., Gordis, E. B., \& Stroud, L. R. (2007). Salivary $\alpha$-amylase in biobehavioural research: Recent developments and applications. Annals of the New York Academy of Sciences, 1098(1), 122-144.

Gunnar, M., \& Quevedo, K. (2007). The neurobiology of stress and development. Annual Review of Psychology, 58(1), 145-173. doi: 10.1146/annurev.psych.58.110405.085605

Gunnar, M. R., Talge, N. M., \& Herrera, A. (2009). Stressor paradigms in developmental studies: What does and does not work to produce mean increases in salivary cortisol. Psychoneuroendocrinology, 34(7), 953-967. doi:10.1016/j.psyneuen.2009.02.010

Gunnar, M. R., \& White, B. P. (2001). Salivary cortisol measures in infant and child assessment. In L. T. Singer, \& P. S. Zeskind (Eds.), Behavioural Assessment of the Infant (pp.953-967). New York, NY: Guildford Press.

Haley, D. W., \& Stansbury, K. (2003). Infant stress and parent responsiveness: Regulation of physiology and behaviour during still-face and reunion. Child Development, 74(5), 15341546.

Hibel, L. C., Granger, D. A., Blair, C., Finegood, E. D., The Family Life Project Key Investigators. (2014). Maternal-child adrenocortical attunement in early childhood: Continuity and change. Developmental Psychobiology, 57, 83-95.

Hill-Soderlund, A. L., Mills-Koonce, W. R., Propper, C., Calkins, S. D., Granger, D. A., Moore, G. A., ..., Cox, M. J. (2008). Parasympathetic and sympathetic responses to the strange situation in infants and mothers from avoidant and securely attached dyads. Developmental Psychobiology, 50, 361-376. doi: 10.1002/dev.20302

Hruschka, D. J., Khort, B. A., \& Worthman, C. M. (2005). Estimating between- and withinindividual variation in cortisol levels using multilevel models. Psychoneuroendocrinology, 30(7), 698-714. doi: 10.1016/j.psyneuen.2005.03.002

Jansen, J., Biejers, R., Riksen-Walraven, M., \& de Weerth, C. (2010). Cortisol reactivity in young infants. Psychoneuroendocrinology, 35, 329-338.

Kendler, K. S., Gardner, C. O., Fiske, A., \& Gatz, M. (2009). Major depression and coronary artery disease in the Swedish twin registry: Phenotypic, genetic, and environmental sources of comorbidity. Archives of General Psychiatry, 66(8), 857-63.

Khoury, J. E., Gonzalez, A., Levitan, R., Masellis, M., Vincenzo, B., \& Atkinson, L. (2016). Infant emotion regulation strategy moderates relations between self-reported maternal 
depressive symptoms and infant HPA activity. Infant and Child Development, 25(1), 64-83. doi: 10.1002/icd.1916

Kirschbaum, C., \& Hellhammer, D. H. (1994). Salivary cortisol in psychoneuroendocrine research: Recent developments and applications. Psychoneuroendocrinology, 19(4), 313333. doi:10.1016/0306-4530(94)90013-2

Kivlighan, K. T. (2006). Dyadic stress vulnerability in mother-infant dyads: Sympathetic nervous system and adrenocortical reactivity to infant challenge. (Doctoral Dissertation). Retrieved from ProQuest. (UMI Number: 3343691).

Kizildere, S., Glück, T., Zietz, B., Schölmerich, J., \& Straub, R. (2003). During a corticotropinreleasing hormone test in healthy subjects, administration of a beta-adrenergic antagonist induced secretion of cortisol and dehydroepiandrosterone sulfate and inhibited secretion of ACTH. European Journal of Endocrinology, 148, 45-53.

Laurent, H. K., Ablow, J. C., \& Measelle, J. (2012). Taking stress response out of the box: Stability, discontinuity, and temperament effects on HPA and SNS across social stressors in Mother-Infant dyads. Developmental Psychology, 48(1), 35-45. doi:10.1037/a0025518

Laurent, H. K., Powers, S. I., \& Granger, D. A. (2013). Refining the multisystem view of the stress response: Coordination among cortisol, alpha-amylase, and subjective stress in response to relationship conflict. Physiology \& Behaviour, 119, 52-60. doi: 10.1016/j.physbeh.2013.05.019

Lazarus, R. S., Speisman, J. C., Mordkoff, A. M. (1963). The relationship between autonomic indicators of psychological stress: Heart rate and skin conductance. Psychosomatic Medicine, 25(1), 19-30.

Letourneau, N., Watson, B., Duffett-Leger, L., Hegadoren, K., \& Tryphonopoulos, P. (2011). Cortisol patterns of depressed mothers and their infants are related to maternal-infant interactive behaviours. Journal of Reproductive and Infant Psychology, 29(5), 439-459. doi:10.1080/02646838.2011.649474

Little, R., \& Rubin, D. (1987). Statistical analysis with missing data. New York, NY: Wiley.

Lovallo, W. R. (2005). Stress and health: Biological and psychological interactions ( $2^{\text {nd }}$ Ed.). Thousand Oaks, CA: Sage Publications.

Ludmer, J. A., Levitan, R., Gonzalez, A., Kennedy, J., Villani, V., Masellis, M., ..., Atkinson, L. (2015). DRD2 and SLC6A3 moderate impact of maternal depressive symptoms on infant cortisol. Psychoneuroendocrinology, 62, 243-251. doi: 10.1016/j.psyneuen.2015.08.026 
Lundberg, U., \& Frankenhaeuser, M. (1980). Pituitary-adrenal and sympathetic-adrenal correlates of distress and effort. Journal of Psychosomatic Research, 24, 125-130.

Mackie, D. A., \& Pangborn, R. M. (1990). Mastication and its influence on human salivary flow and alpha-amylase secretion. Physiology \& Behaviour, 47, 593-595.

McCoach, D. B., \& Black, A. C. (2008). Assessing model adequacy. In Ann A. O'Connell and D. Betsy McCoach (Eds.) Multilevel modeling of educational data (pp. 245-272). Charlotte, NC: Information Age Publishing.

McEwen, B. S., \& Gianaros, P. J. (2011). Stress- and allostasis-induced brain plasticity. Annual Review of Medicine, 62, 431-435. doi: 10.1146/annurev-med-052209-100430.

McEwen, B. S., \& Seeman, T. (1999). Protective and damaging effects of mediators of stress: Elaborating and testing concepts of allostasis and allostatic load. Annals of the New York Academy of Science, 896(1), 30-47.

McEwen, B. S., \& Wingfield, J. C. (2003). The concept of allostasis in biology and biomedicine. Hormones and Behaviour, 43, 2-15.

Meaney, M. J., \& Szyf, M. (2005). Environmental programming of stress responses through DNA methylation: life at the interface between a dynamic environment and a fixed genome. Dialogues in Clinical Neuroscience, 7(2), 103-123.

Miller, G. E., Chen, E., \& Zhou, E. S. (2007). If it goes up, must it come down? Chronic stress and the hypothalamic-pituitary-adrenocortical axis in humans. Psychological Bulletin, 133(1), 25-45. doi: 10.1037/0033-2909.133.1.25

Munck, A., Guyre, P. M., \& Holbrook, N. J. (1984). Psychological functions of glucocorticoids in stress and their relation to pharmacological actions. Endocrine Reviews, 5, 25-43.

Nachmias, M., Gunnar, M., Mangelsdorf, S., Hornik Parritz, R., \& Bus, K. (1996). Behavioural inhibition and stress reactivity: The moderating role of attachment security. Child Development, 67, 508-522.

Nater, U. M., La Marca, R., Florin, L., Moses, A., Langhans, W., Koller, M. M., Ehlert, U. (2006). Stress-induced changes in human salivary alpha-amylase activity - associations with adrenergic activity. Psychneuroendocrinology, 31, 49-58.

Nater, U. M., \& Rohleder, N. (2009). Salivary alpha-amylase as a non-invasive biomarker for the sympathetic nervous system: Current state of research. Psychoneuroendocrinology, 34(4), 486-496. doi:10.1016/j.psyneuen.2009.01.014 
Nater, U. M., Rohleder, N., Gaab, J., Berger, S., Jud. A., Kirschbaum, C., \& Ehlert, U. (2005). Human salivary alpha-amylase reactivity in a psychosocial stress paradigm. International Journal of Psychophysiology, 55, 333-342.

Newlin, D. B., \& Levenson, R. W. (1979). Pre-ejection period: Measuring beta-adrenergic influences upon the heart. Psychophysiology, 16(6), 546-553.

O'Donnell, M. D., \& Miller, N. J. (1980). Plasma pancreatic and salivary-type amylase and immunoreactive trypsin concentrations: variations with age and reference ranges for children. Clinica Chimica Acta, 104, 265-273.

O'Keane, V., Frodl, T., \& Dinan, T. G. (2012). A review of atypical depression in relation to the course of depression and changes in HPA axis organization. Psychoneuroendocrinology, 37, 1589-1599. doi:10.1016/j.psyneuen.2012.03.009

Oei, N. Y. L., Tollenaar, M. S., Elzinga, B. M., \& Spinhoven, P. (2010). Propranolol reduces emotional distraction in working memory: A partial mediating role of propranolol-induced cortisol increases? Neurobiology of Learning and Memory, 93, 388-395.

doi:10.1016/j.nlm.2009.12.005

Pederson, D. R., Moran, G., Sitko, C., Campbell, K., Ghesquire, K., \& Acton, H. (1990). Maternal sensitivity and the security of infant-mother attachment: A Q-sort study. Child Development, 61, 1974-1983.

Pereira, J., Vickers, K., Atkinson, L., Gonzalez, A., Wekerle, C., \& Levitan, R. (2012). Parenting stress mediates between maternal maltreatment history and maternal sensitivity in a community sample. Child Abuse \& Neglect, 36(5), 433-437.

Preacher, K. J., Curran, P. J., \& Bauer, D. J. (2006). Computational tools for probing interaction effects in multiple linear regression, multilevel modeling, and latent curve analysis. Journal of Educational and Behavioral Statistics, 31, 437-448.

Raudenbush, S., Bryk, A., Cheong, Y. F., Congdon, R., du Toit, M. (2011). HLM 7 Hierarchical linear and nonlinear modelling. Lincolnwood, IL: Scientific Software International.

Rohleder, N., Nater, U. M., Wolf, J. M., Ehlert, U., \& Kirschbaum, C. (2004). Psychosocial stress-induced activation of salivary alpha-amylase: an indicator of sympathetic activity? Annals of the New York Academy of Sciences, 1032, 258-263.

Sakaue, M., \& Hoffman, B. B. (1991). Glucocorticoids induce transcription and expression of the $\alpha 1 \mathrm{~B}$ adrenergic receptor gene in DTTI MF-2 smooth muscle cells. Journal of Clinical Investigation, 88, 385-389. 
Sapolsky, R. M., Romero, L. M., \& Munck, A .U. (2000). How do glucocorticoids influence stress responses? Integrating permissive, suppressive, stimulatory, and preparative actions. Endocrine Reviews, 21(1), 55-89.

Scannapieco, F. A., Torres, G., \& Levine, M.J. (1993). Salivary alpha-amylase: role in dental plaque and caries formation. Critical Review of Oral Biology, 4, 301-307.

Schafer, J. L., \& Olsen, M. K. (1998). Multiple imputation for multivariate missing-data problems: A data analyst's perspective. Multivariate Behavioural Research, 33(4), 545-571.

Schommer, N. C., Hellhammer, D. H., \& Kirschbaum, C. (2003). Dissociation between reactivity of the hypothalamus-pituitary-adrenal axis and the sympathetic-adrenal-medullary system to repeated psychosocial stress. Psychosomatic Medicine, 65, 450-460. doi: 10.1097/01.PSY.0000035721.12441.17

Singer, J. D., \& Willett, J. B. (2003). Applied longitudinal data analysis: Modeling change and event occurrence. New York, NY: Oxford University Press.

Skoluda, N., Strahler, J., Schlotz, W., Niederberger, L., Margues, S., Fischer, S., ... Nater, U. M. (2015). Intra-individual psychological and physiological responses to acute laboratory stressors of different intensity. Psychoneuroendocrinology, 51, 227-236. doi: 10.1016/j.psyneuen.2014.10.002

Spanglar, G., Schieche, M., Ilg, U., Maier, U., \& Ackermann, C. (1994). Maternal sensitivity as an external organizer for biobehavioral regulation in infancy. Developmental Psychobiology, 27(7), 425-437. doi:10.1002/dev.420270702

Spinrad, T. L., Eisenberg, N., Granger, D. A., Eggum, N. D., Sallquist, J., Haugen, R. G., ... Hofer, C. (2009). Individual differences in preschoolers' salivary cortisol and alpha-amylase reactivity: relations to temperament and maladjustment. Hormones \& Behavior, 56, 133139.

Steptoe, A., \& Kivimäki, M. (2012). Stress and cardiovascular disease. Nature Reviews Cardiology, 9(6), 360-370. doi:10.1038/nrcardio.2012.45

Stroud, L. R., Foster, E., Papandonatos, G. D., Handwerger, K., Granger, D. A., Kivlighan, K. T., \& Niaura, R. (2009). Stress response and the adolescent transition: performance versus peer rejection stressors. Developmental Psychopathology, 21(1), 47-68.

Takai, N., Yamaguchi, M., Aragaki, T., Eto, K., Uchihashi, K., \& Nishikawa, Y. (2007). Genderspecific differences in salivary biomarker responses to acute psychological stress. Annals of the New York Academy of Sciences, 1098(1), 510-515. 
Tarullo, A. R., \& Gunnar, M. R. (2006). Child maltreatment and the developing HPA axis. Hormones and Behavior, 50(4), 632-639. doi:10.1016/j.yhbeh.2006.06.010

Taylor, S. E., Klein, L. C., Lewis, B. P., Gruenewald, T. L., Gurung, R. A. R., \& Updegraff, J. A. (2000). Biobehavioral responses to stress in females: Tend-and-befriend, not fight-or-flight. Psychological Review, 107, 411-429.

Thompson, L. A., Trevathan, W. R. (2009). Cortisol reactivity, maternal sensitivity, and learning in three-month-old infants. Infant Behaviour and Development, 31, 92-106.

Ulrich-Lai, Y. M., \& Herman, J. P. (2009). Neural regulation of endocrine and autonomic stress responses. Nature Reviews: Neuroscience, 10, 397-409.

Ursin, H., Baade, E., \& Levine, S. (1978). Psychobiology of Stress. New York, NY: Academic Press.

van Bakel, H. J., \& Risken-Walraven, J. M. (2004). Stress reactivity in 15-month-old infants: links with temperament, cognitive competence and attachment security. Developmental Psychobiology, 44, 157-167.

Vigil, J. M. (2009). A socio-relational framework of sex differences in the expression of emotion. Behavioral and Brain Sciences, 32, 375-390.

Vigil, J. M., Geary, D. C., Granger, D. A., \& Flinn, M. V. (2010). Sex differences in salivary cortisol, alpha-amylase, and psychological functioning after Hurricane Katrina. Child Development, $81,1228-1240$.

Villani, V. C., \& Jamieson, B. (in prep). Salivary alpha-amylase reactivity to acute laboratory stressors: A meta-analytic review.

Viru, A., Viru, M., Karelson, K., Janson, T., Siim, K., Fischer, K., \& Hackney, A. C. (2007). Adrenergic effects on adrenocortical cortisol response to incremental exercise to exhaustion. European Journal of Applied Physiology, 100, 241-245. doi: 10.1007/s00421-007-0416-9

Williams, R. B., Marchuk, D. A., Siegler, I. C., Barefoot, J. C., Helms, M. J., Brummett, B. H., ..., Schanberg, S. M. (2008). Childhood socioeconomic status and serotonin transporter gene polymorphism enhance cardiovascular reactivity to mental stress. Psychosomatic Medicine, 70, 32-29. doi: 10.1097/PSY.0b013e31815f66c3

Willett, J. B., \& Sayer, A. G. (1994). Using covariance structure analysis to detect correlates and predictors of individual change over time. Psychological Bulletin, 116(2), 363-381. 